\title{
A Parabolic Quasilinear Problem for Linear Growth Functionals
}

Fuensanta Andreu, Vincent Caselles and José María Mazón

Dedicated to Philippe Bénilan on the Occasion of His 60th Birthday

\begin{abstract}
We prove existence and uniqueness of solutions for the Dirichlet problem for quasilinear parabolic equations in divergent form for which the energy functional has linear growth. A typical example of energy functional we consider is the one given by the nonparametric area integrand $f(x, \xi)=\sqrt{1+\|\xi\|^{2}}$, which corresponds with the time-dependent minimal surface equation. We also study the asymptotic behaviour of the solutions.
\end{abstract}

\section{Introduction and preliminaries}

Let $\Omega$ be a bounded set in $\mathbb{R}^{N}$ with Lipschitz continuous boundary $\partial \Omega$. We are interested in the problem

$$
\begin{cases}\frac{\partial u}{\partial t}=\operatorname{div} \mathbf{a}(x, D u) & \text { in } \quad Q=(0, \infty) \times \Omega, \\ u(t, x)=\varphi(x) & \text { on } \quad S=(0, \infty) \times \partial \Omega, \\ u(0, x)=u_{0}(x) & \text { in } \quad x \in \Omega,\end{cases}
$$

where $u_{0} \in L^{2}(\Omega)$ and $\mathbf{a}(x, \xi)=\nabla_{\xi} f(x, \xi), f$ being a function with linear growth as $\|\xi\| \rightarrow \infty$.

A typical example of a function $f(x, \xi)$ satisfying the conditions we need is the nonparametric area integrand $f(x, \xi)=\sqrt{1+\|\xi\|^{2}}$. Problem (1.1) for this particular $f$, that is, the time-dependent minimal surface equation,

2000 Mathematics Subject Classification: 35K65, 35K55, 47H06, 47H20

Keywords: Linear growth functionals, nonlinear parabolic equations, accretive operators, nonlinear semigroups 
has been studied in [12] and [17]. Another examples of problems of type (1.1) included in our case, are the following: The evolution problem for plastic antiplanar shear, studied in [21], which corresponds to the plasticity functional $f$ given by

$$
f(\xi)= \begin{cases}\frac{1}{2}\|\xi\|^{2} & \text { if } \quad\|\xi\| \leq 1 \\ \|\xi\|-\frac{1}{2} & \text { if } \quad\|\xi\| \geq 1\end{cases}
$$

and the evolution problems associated with the Lagrangians:

$$
f(x, \xi)=\sqrt{1+a_{i j}(x) \xi_{i} \xi_{j}},
$$

where the functions $a_{i j}$ are continuous and satisfy $a_{i j}(x)=a_{j i}(x),\|\xi\|^{2} \leq$ $a_{i j}(x) \xi_{i} \xi_{j} \leq C\|\xi\|^{2}$ for all $\xi \in \mathbb{R}^{N}$; and the Lagrangian

$$
g(x, \xi)=\sqrt{1+x^{2}+\|\xi\|^{2}}
$$

which was considered by S. Bernstein ([8]). On the other hand, problem (1.1) is studied in [14] for some Lagrangians $f$, which do not include the nonparametric area integrand, but instead include the plasticity functional and the total variation flow, that is, the case $f(\xi)=\|\xi\|$. Now, the concept of solution given in [14] is the one obtained by considering the abstract Cauchy problem in $L^{2}(\Omega)$ associated to the relaxed energy, but the subdifferential of the energy functional is not characterized. For the particular case of the total variation flow, we give in [4] a different approach to the Dirichlet problem. There, we studied the problem in the framework of the $L^{1}$ - theory, and we characterized the subdifferential in $L^{2}(\Omega)$ of its relaxed energy (we refer also to [3] where we treated the $L^{1}$-theory for the Neumann problem for the total variational flow).

In general, problem (1.1) does not have a classical solution. The aim of this paper is to introduce a concept of solution of the Dirichlet problem (1.1), for which existence and uniqueness for initial data in $L^{2}(\Omega)$ is proved. To do that we characterize the subdifferential of the energy associated with the problem and we use the nonlinear semigroup theory. In a forthcoming paper we will study the same problem in the framework of the $L^{1}$-theory, as we did with the Dirichlet problem for the total variational flow ([4]).

In order to consider the relaxed energy we recall the definition of function of measure (see for instance, [6] or [12]). Let $g: \Omega \times \mathbb{R}^{N} \rightarrow \mathbb{R}$ be a Carathéodory function such that

$$
|g(x, \xi)| \leq M(1+\|\xi\|) \quad \forall(x, \xi) \in \Omega \times \mathbb{R}^{N},
$$


for some constant $M \geq 0$. Furthermore, we assume that $g$ possesses an asymptotic function, i.e. for almost all $x \in \Omega$ there exists the finite limit

$$
\lim _{t \rightarrow 0^{+}} \operatorname{tg}\left(x, \frac{\xi}{t}\right)=g^{0}(x, \xi)
$$

It is clear that the function $g^{0}(x, \xi)$ is positively homogeneous of degree one in $\xi$, i.e.

$$
g^{0}(x, s \xi)=s g^{0}(x, \xi) \quad \text { for all } x, \xi \text { and } s>0 .
$$

We denote by $\mathcal{M}\left(\Omega, \mathbb{R}^{N}\right)$ the set of all $\mathbb{R}^{N}$-valued bounded Radon measures on $\Omega$. Given $\mu \in \mathcal{M}\left(\Omega, \mathbb{R}^{N}\right)$, we consider its Lebesgue decomposition

$$
\mu=\mu^{a}+\mu^{s},
$$

where $\mu^{a}$ is the absolutely continuous part of $\mu$ with respect to the Lebesgue measure $\lambda_{N}$ of $\mathbb{R}^{N}$, and $\mu^{s}$ is singular with respect to $\lambda_{N}$. We denote by $\mu^{a}(x)$ the density of the measure $\mu^{a}$ with respect to $\lambda_{N}$ and by $\left(d \mu^{s} / d|\mu|^{s}\right)(x)$ the density of $\mu^{s}$ with respect to $|\mu|^{s}$.

Given $\mu \in \mathcal{M}\left(\Omega, \mathbb{R}^{N}\right)$, we define $\tilde{\mu} \in \mathcal{M}\left(\Omega, \mathbb{R}^{N+1}\right)$ by

$$
\tilde{\mu}(B):=\left(\mu(B), \lambda_{N}(B)\right)
$$

for every Borel set $B \subset \mathbb{R}^{N}$. Then, we have

$$
\tilde{\mu}=\tilde{\mu}^{a}+\tilde{\mu}^{s}=\tilde{\mu}^{a}(x) \lambda_{N}+\tilde{\mu}^{s}=\left(\mu^{a}(x), \mathbf{1}\right) \lambda_{N}+\left(\mu^{s}, 0\right) .
$$

Hence, we have

$$
\left|\tilde{\mu}^{s}\right|=\left|\mu^{s}\right|, \quad \frac{d \tilde{\mu}^{s}}{d\left|\tilde{\mu}^{s}\right|}=\left(\frac{d \mu^{s}}{d\left|\mu^{s}\right|}, 0\right) \quad\left|\mu^{s}\right| \text { - a.e. }
$$

For $\mu \in \mathcal{M}\left(\Omega, \mathbb{R}^{N}\right)$ and $g$ satisfying the above conditions, we define the measure $g(x, \mu)$ on $\Omega$ as

$$
\int_{B} g(x, \mu):=\int_{B} g\left(x, \mu^{a}(x)\right) d x+\int_{B} g^{0}\left(x, \frac{d \mu^{s}}{d|\mu|^{s}}(x)\right) d|\mu|^{s}
$$

for all Borel set $B \subset \Omega$. In formula (1.4) we may write $(d \mu / d|\mu|)(x)$ instead of $\left(d \mu^{s} / d|\mu|^{s}\right)(x)$, because the two functions are equal $|\mu|^{s}$-a.e.

Another way of writing the measure $g(x, \mu)$ is the following. Let us consider the function $\tilde{g}: \Omega \times \mathbb{R}^{N} \times[0,+\infty[\longrightarrow \mathbb{R}$ defined as

$$
\tilde{g}(x, \xi, t):=\left\{\begin{array}{lll}
g\left(x, \frac{\xi}{t}\right) t & \text { if } & t>0 \\
g^{0}(x, \xi) & \text { if } & t=0 .
\end{array}\right.
$$


As it is proved in [6], if $g$ is a Carathéodory function satisfying (1.2), then one has

$$
\int_{B} g(x, \mu)=\int_{B} \tilde{g}\left(x, \frac{d \mu}{d \alpha}(x), \frac{d \lambda_{N}}{d \alpha}(x)\right) d \alpha
$$

where $\alpha$ is any positive Borel measure such that $|\mu|+\lambda_{N} \ll \alpha$.

Due to the linear growth condition on the Lagrangian, the natural energy space to study (1.1) is the space of functions of bounded variation. Let us recall several facts concerning functions of bounded variation (for further information concerning functions of bounded variation we refer to [13], [22] or $[2])$.

A function $u \in L^{1}(\Omega)$ whose partial derivatives in the sense of distributions are measures with finite total variation in $\Omega$ is called a function of bounded variation. The class of such functions will be denoted by $B V(\Omega)$. Thus $u \in B V(\Omega)$ if and only if there are Radon measures $\mu_{1}, \ldots, \mu_{N}$ defined in $\Omega$ with finite total mass in $\Omega$ and

$$
\int_{\Omega} u D_{i} \varphi d x=-\int_{\Omega} \varphi d \mu_{i}
$$

for all $\varphi \in C_{0}^{\infty}(\Omega), i=1, \ldots, N$. Thus the gradient of $u$ is a vector valued measure with finite total variation

$$
\|D u\|=\sup \left\{\int_{\Omega} u \operatorname{div} \varphi d x: \varphi \in C_{0}^{\infty}\left(\Omega, \mathbb{R}^{N}\right),|\varphi(x)| \leq 1 \text { for } x \in \Omega\right\} .
$$

The space $B V(\Omega)$ is endowed with the norm

$$
\|u\|_{B V}=\|u\|_{L^{1}(\Omega)}+\|D u\| .
$$

For $u \in B V(\Omega)$, the gradient $D u$ is a Radon measure that decomposes into its absolutely continuous and singular parts $D u=D^{a} u+D^{s} u$. Then $D^{a} u=$ $\nabla u \lambda_{N}$ where $\nabla u$ is the Radon-Nikodym derivative of the measure $D u$ with respect to the Lebesgue measure $\lambda_{N}$. There is also the polar decomposition $D^{s} u=\overrightarrow{D^{s} u}\left|D^{s} u\right|$ where $\left|D^{s} u\right|$ is the total variation measure of $D^{s} u$.

We shall need several results from [5] (see also [16]). Following [5], let

$$
X(\Omega)=\left\{z \in L^{\infty}\left(\Omega, \mathbb{R}^{N}\right): \operatorname{div}(z) \in L^{1}(\Omega)\right\} .
$$

If $z \in X(\Omega)$ and $w \in B V(\Omega) \cap L^{\infty}(\Omega)$ we define the functional $(z, D w)$ : $C_{0}^{\infty}(\Omega) \rightarrow \mathbb{R}$ by the formula

$$
<(z, D w), \varphi>=-\int_{\Omega} w \varphi \operatorname{div}(z) d x-\int_{\Omega} w z \cdot \nabla \varphi d x .
$$


Then $(z, D w)$ is a Radon measure in $\Omega$,

$$
\int_{\Omega}(z, D w)=\int_{\Omega} z \cdot \nabla w d x \quad \forall w \in W^{1,1}(\Omega) \cap L^{\infty}(\Omega)
$$

and

$$
\left|\int_{B}(z, D w)\right| \leq \int_{B}|(z, D w)| \leq\|z\|_{\infty} \int_{B}\|D w\|
$$

for any Borel set $B \subseteq \Omega$. Moreover, $(z, D w)$ is absolutely continuous with respect to $\|D w\|$ with Radon-Nikodym derivative $\theta(z, D w, x)$ which is a $\|D w\|$ measurable function from $\Omega$ to $\mathbb{R}$ such that

$$
\int_{B}(z, D w)=\int_{B} \theta(z, D w, x)\|D w\|
$$

for any Borel set $B \subseteq \Omega$. We also have that

$$
\|\theta(z, D w, .)\|_{L^{\infty}(\Omega,\|D w\|)} \leq\|z\|_{L^{\infty}\left(\Omega, \mathbb{R}^{N}\right)} .
$$

By writing

$$
z \cdot D^{s} u:=(z, D u)-(z \cdot \nabla u) d \lambda_{N},
$$

we see that $z \cdot D^{s} u$ is a bounded measure. Furthermore, in [16] it is proved that $z \cdot D^{s} u$ is absolutely continuous with respect to $\left|D^{s} u\right|$ (and, thus, it is a singular measure with respect to $\lambda_{N}$ ), and

$$
\left|z \cdot D^{s} u\right| \leq\|z\|_{\infty}\left|D^{s} u\right| .
$$

As a consequence of Theorem 2.4 of [5], we have:

$$
\text { If } z \in X(\Omega) \cap C\left(\Omega, \mathbb{R}^{N}\right) \text {, then } z \cdot D^{s} u=\left(z \cdot \overrightarrow{D^{s} u}\right) d\left|D^{s} u\right| \text {. }
$$

In [5], a weak trace on $\partial \Omega$ of the normal component of $z \in X(\Omega)$ is defined. Concretely, it is proved that there exists a linear operator $\gamma$ : $X(\Omega) \rightarrow L^{\infty}(\partial \Omega)$ such that

$$
\begin{gathered}
\|\gamma(z)\|_{\infty} \leq\|z\|_{\infty} \\
\gamma(z)(x)=z(x) \cdot \nu(x) \quad \text { for all } x \in \partial \Omega \text { if } z \in C^{1}\left(\bar{\Omega}, \mathbb{R}^{N}\right) .
\end{gathered}
$$

We shall denote $\gamma(z)(x)$ by $[z, \nu](x)$. Moreover, the following Green's formula, relating the function $[z, \nu]$ and the measure $(z, D w)$, for $z \in X(\Omega)$ and $w \in B V(\Omega) \cap L^{\infty}(\Omega)$, is established:

$$
\int_{\Omega} w \operatorname{div}(z) d x+\int_{\Omega}(z, D w)=\int_{\partial \Omega}[z, \nu] w d H^{N-1}
$$


Let $g$ be a function satisfying (1.2). Then for every $u \in B V(\Omega)$ we have the measure $g(x, D u)$ defined by

$$
\int_{B} g(x, D u)=\int_{B} g(x, \nabla u(x)) d x+\int_{B} g^{0}\left(x, \overrightarrow{D^{s} u}(x)\right) d\left|D^{s} u\right|
$$

for all Borel set $B \subset \Omega$. If we assume that $\Omega$ has a Lipschitz boundary, and that $g(x, \xi)$ is defined also for $x \in \partial \Omega$, we may consider the functional $G$ in $B V(\Omega)$ defined by

$$
G(u):=\int_{\Omega} g(x, D u)+\int_{\partial \Omega} g^{0}(x, \nu(x)[\varphi(x)-u(x)]) d H^{N-1}
$$

where $\varphi \in L^{1}(\partial \Omega)$ is a given function and $\nu$ is the outer unit normal to $\partial \Omega$. It is proved in [6] that, if $\tilde{g}(x, \xi, t)$ is continuous on $\bar{\Omega} \times \mathbb{R}^{N} \times[0,+\infty[$ and convex in $(\xi, t)$ for each fixed $x \in \bar{\Omega}$, then $G$ is the greatest functional on $B V(\Omega)$ which is lower-semicontinuous with respect to the $L^{1}(\Omega)$-convergence and satisfies

$$
G(u) \leq \int_{\Omega} g(x, \nabla u(x)) d x
$$

for all functions $u \in C^{1}(\Omega) \cap W^{1,1}(\Omega)$ with $u=\varphi$ on $\partial \Omega$.

The paper is organized as follows: in Section 2 we give the definition of solution for the Dirichlet problem and we state the existence and uniqueness result for this type of solutions. Section 3 is devoted to prove the existence and uniqueness result. To do that, we study the problem from the point of view of nonlinear semigroup theory. We characterize the subdifferential in $L^{2}(\Omega)$ of the relaxed energy functional associated with the problem. In Section 4 we give a weakened form of the maximum principle and we study the asymptotic behaviour of solutions proving that they stabilize as $t \rightarrow \infty$ by converging to a solution of the steady-state problem. Finally, the Appendix contains the proof of the approximation Lemma stated in Section 3.

\section{The existence and uniqueness result}

In this section we define the concept of solution for the Dirichlet problem (1.1) and we state the existence and uniqueness result for this type of solutions when the initial data are in $L^{2}(\Omega)$.

Here we assume that $\Omega$ is an open bounded set in $\mathbb{R}^{N}, N \geq 2$, with boundary $\partial \Omega$ of class $C^{1}$, and the Lagrangian $f: \bar{\Omega} \times \mathbb{R}^{N} \rightarrow \mathbb{R}$ satisfies the following assumptions, which we shall refer collectively as $(\mathrm{H})$ : 
$\left(\mathrm{H}_{1}\right) \quad f$ is continuous on $\bar{\Omega} \times \mathbb{R}^{N}$ and is a convex diffentiable function of $\xi$ with continuous gradient for each fixed $x \in \Omega$. Further we require $f$ to satisfy the linear growth condition

$$
C_{0}\|\xi\|-C_{1} \leq f(x, \xi) \leq M\left(\|\xi\|+C_{2}\right) .
$$

for some positive constants $C_{0}, C_{1}, C_{2}$. Moreover, $f^{0}$ exists and $f^{0}(x,-\xi)=f^{0}(x, \xi)$ for all $\xi \in \mathbb{R}^{N}$ and all $x \in \bar{\Omega}$.

$\left(\mathrm{H}_{2}\right) \tilde{f}(x, \xi, t)$ is continuous on $\bar{\Omega} \times \mathbb{R}^{N} \times[0,+\infty[$ and convex in $(\xi, t)$ for each fixed $x \in \bar{\Omega}$.

We consider the function $\mathbf{a}(x, \xi)=\nabla_{\xi} f(x, \xi)$ associated to the Lagrangian $f$. By the convexity of $f$

$$
\mathbf{a}(x, \xi) \cdot(\eta-\xi) \leq f(x, \eta)-f(x, \xi)
$$

and the following monotonicity condition is satisfied

$$
(\mathbf{a}(x, \eta)-\mathbf{a}(x, \xi)) \cdot(\eta-\xi) \geq 0 .
$$

Moreover, it is easy to see that

$$
|\mathbf{a}(x, \xi)| \leq M \quad \forall(x, \xi) \in \Omega \times \mathbb{R}^{N} .
$$

We consider the function $h: \Omega \times \mathbb{R}^{N} \rightarrow \mathbb{R}$ defined by

$$
h(x, \xi):=\mathbf{a}(x, \xi) \cdot \xi .
$$

From (2.2) and (2.1), it follows that

$$
C_{0}\|\xi\|-D_{1} \leq h(x, \xi) \leq M\|\xi\|
$$

for some positive constant $D_{1}$.

We assume that

$\left(\mathrm{H}_{3}\right) h(x, \xi) \geq 0$ for all $x \in \Omega$ and $\xi \in \mathbb{R}^{N}, h^{0}$ exists and the function $\tilde{h}$ is continuous on $\bar{\Omega} \times \mathbb{R}^{N} \times[0,+\infty[$.

We need to consider the mapping $\mathbf{a}^{\infty}$ defined by

$$
\mathbf{a}^{\infty}(x, \xi):=\lim _{t \rightarrow+\infty} \mathbf{a}(x, t \xi) .
$$

Observe that

$$
h^{0}(x, \xi)=\mathbf{a}^{\infty}(x, \xi) \cdot \xi \quad \text { and } \quad C_{0}\|\xi\| \leq h^{0}(x, \xi) \leq M\|\xi\| .
$$


$\left(\mathrm{H}_{4}\right) \mathbf{a}^{\infty}(x, \xi)=\nabla_{\xi} f^{0}(x, \xi)$ for all $\xi \neq 0$ and all $x \in \bar{\Omega}$.

In particular, as a consequence of Euler's Theorem, we have

$$
f^{0}(x, \xi)=\mathbf{a}^{\infty}(x, \xi) \cdot \xi=h^{0}(x, \xi),
$$

for all $\xi \in \mathbb{R}^{N}$ and all $x \in \bar{\Omega}$.

$\left(\mathrm{H}_{5}\right) \mathbf{a}(x, \xi) \cdot \eta \leq h^{0}(x, \eta)$ for all $\xi, \eta \in \mathbb{R}^{N}$, and all $x \in \bar{\Omega}$.

Either from $\left(\mathrm{H}_{4}\right)$ or $\left(\mathrm{H}_{5}\right)$ it follows that $\mathbf{a}^{\infty}(x, \xi) \cdot \eta \leq h^{0}(x, \eta)$ for all $\xi, \eta \in$ $\mathbb{R}^{N}, \xi \neq 0$, and all $x \in \bar{\Omega}$. Indeed, it suffices to replace $\xi$ by $t \xi$ in $\left(\mathrm{H}_{5}\right)$ and let $t \rightarrow+\infty$.

Definition 2.1 Let $\varphi \in L^{1}(\partial \Omega)$ and $u_{0} \in L^{2}(\Omega)$. A measurable function $u$ : $(0, T) \times \Omega \rightarrow \mathbb{R}$ is a solution of $(1.1)$ in $Q_{T}=(0, T) \times \Omega$ if $u \in C\left([0, T], L^{2}(\Omega)\right)$, $u(0)=u_{0}, u^{\prime}(t) \in L^{2}(\Omega), u(t) \in B V(\Omega) \cap L^{2}(\Omega), \mathbf{a}(x, \nabla u(t)) \in X(\Omega)$ a.e. $t \in[0, T]$, and for almost all $t \in[0, T] u(t)$ satisfies:

$$
\begin{gathered}
u^{\prime}(t)=\operatorname{div}\left(\mathbf{a}(x, \nabla u(t)) \quad \text { in } \mathcal{D}^{\prime}(\Omega)\right. \\
\mathbf{a}(x, \nabla u(t)) \cdot D^{s} u(t)=f^{0}\left(x, D^{s} u(t)\right) \\
{[\mathbf{a}(x, \nabla u(t)), \nu] \in \operatorname{sign}(\varphi-u(t)) f^{0}(x, \nu(x)) \quad H^{N-1}-a . e . \text { on } \partial \Omega .}
\end{gathered}
$$

Our main result is the following:

Theorem 2.2 Let $\varphi \in L^{1}(\partial \Omega)$ and assume we are under assumptions (H). Given $u_{0} \in L^{2}(\Omega)$, there exists a unique solution u of (1.1) in $Q_{T}$ for every $T>0$ such that $u(0)=u_{0}$.

\section{Strong solution for data in $L^{2}(\Omega)$}

To prove Theorem 2.2 we shall use the nonlinear semigroup theory ([9]). For $\varphi \in L^{1}(\partial \Omega)$ we define the energy functional associated with the problem $(1.1) \Phi_{\varphi}: L^{2}(\Omega) \rightarrow[0,+\infty]$ by

$$
\Phi_{\varphi}(u):=\int_{\Omega} f(x, D u)+\int_{\partial \Omega} f^{0}(x, \nu(x)[\varphi-u]) d H^{N-1}
$$

if $u \in B V(\Omega) \cap L^{2}(\Omega)$ and

$$
\Phi_{\varphi}(u):=+\infty \quad \text { if } u \in L^{2}(\Omega) \backslash B V(\Omega) .
$$


Note that, on the boundary, the integrand can be written in the form

$$
f^{0}(x, \nu(x)[\varphi-u])=|\varphi-u| f^{0}(x, \nu(x)) .
$$

Functional $\Phi_{\varphi}$ is clearly convex and has the form given in (1.19). Then, as a consequence of the Anzellotti's result ([6]) we have that $\Phi_{\varphi}$ is lowersemicontinuous. Therefore, the subdifferential $\partial \Phi_{\varphi}$ of $\Phi_{\varphi}$, i.e. the operator in $L^{2}(\Omega)$ defined by

$$
v \in \partial \Phi_{\varphi}(u) \Longleftrightarrow \Phi_{\varphi}(w)-\Phi_{\varphi}(u) \geq \int_{\Omega} v(w-u) d x, \quad \forall w \in L^{2}(\Omega)
$$

is a maximal monotone operator in $L^{2}(\Omega)$. Consequently, the existence and uniqueness of a solution of the abstract Cauchy problem

$$
\begin{cases}u^{\prime}(t)+\partial \Phi_{\varphi}(u(t)) \ni 0 & t \in] 0, \infty[ \\ u(0)=u_{0} & u_{0} \in L^{2}(\Omega)\end{cases}
$$

follows immediately from the nonlinear semigroup theory (see [9]). Now, to get the full strength of the abstract result derived from semigroup theory we need to characterize $\partial \Phi_{\varphi}$. To get this characterization, we introduce the following operator $\mathcal{B}_{\varphi}$ in $L^{2}(\Omega) . \quad(u, v) \in \mathcal{B}_{\varphi} \Longleftrightarrow u \in B V(\Omega) \cap L^{2}(\Omega)$, $v \in L^{2}(\Omega)$ and $a(x, \nabla u) \in X(\Omega)$ satisfies:

$$
\begin{gathered}
-v=\operatorname{div} \mathbf{a}(x, \nabla u) \quad \text { in } \mathcal{D}^{\prime}(\Omega) \\
\mathbf{a}(x, \nabla u) \cdot D^{s} u=f^{0}\left(x, D^{s} u\right)=f^{0}\left(x, \overrightarrow{D^{s} u}\right)\left|D^{s} u\right|, \\
{[\mathbf{a}(x, \nabla u), \nu] \in \operatorname{sign}(\varphi-u) f^{0}(x, \nu(x)) \quad H^{N-1}-\text { a.e.. }}
\end{gathered}
$$

Let $(u, v) \in \mathcal{B}_{\varphi}$, and $w \in B V(\Omega) \cap L^{2}(\Omega)$. Multiplying (3.2) by $w-u$, and using Green's formula (1.18), we obtain

$$
\begin{gathered}
\int_{\Omega}(w-u) v d x=-\int_{\Omega}(w-u) d i v \mathbf{a}(x, \nabla u) d x= \\
\int_{\Omega}(\mathbf{a}(x, \nabla u), D w-D u)-\int_{\partial \Omega}[\mathbf{a}(x, \nabla u), \nu](w-u) d H^{N-1}= \\
=\int_{\Omega}(\mathbf{a}(x, \nabla u), D w)-\int_{\partial \Omega}[\mathbf{a}(x, \nabla u), \nu](w-\varphi) d H^{N-1}- \\
-\int_{\Omega}(\mathbf{a}(x, \nabla u), D u)-\int_{\partial \Omega}[\mathbf{a}(x, \nabla u), \nu](\varphi-u) d H^{N-1}=
\end{gathered}
$$




$$
\begin{gathered}
=\int_{\Omega}(\mathbf{a}(x, \nabla u), D w)-\int_{\partial \Omega}[\mathbf{a}(x, \nabla u), \nu](w-\varphi) d H^{N-1}- \\
-\int_{\Omega} \mathbf{a}(x, \nabla u) \cdot \nabla u d x-\int_{\Omega} \mathbf{a}(x, \nabla u) \cdot D^{s} u-\int_{\partial \Omega}|\varphi-u| f^{0}(x, \nu(x)) d H^{N-1}= \\
=\int_{\Omega}(\mathbf{a}(x, \nabla u), D w)-\int_{\partial \Omega}[\mathbf{a}(x, \nabla u), \nu](w-\varphi) d H^{N-1}- \\
-\int_{\Omega} h(x, D u)-\int_{\partial \Omega}|\varphi-u| f^{0}(x, \nu(x)) d H^{N-1} .
\end{gathered}
$$

Therefore, if $(u, v) \in \mathcal{B}_{\varphi}$, we have that

$$
\begin{aligned}
\int_{\Omega}(w-u) v d x= & \int_{\Omega}(\mathbf{a}(x, \nabla u), D w)-\int_{\partial \Omega}[\mathbf{a}(x, \nabla u), \nu](w-\varphi) d H^{N-1} \\
& -\int_{\Omega} h(x, D u)-\int_{\partial \Omega}|\varphi-u| f^{0}(x, \nu(x)) d H^{N-1}
\end{aligned}
$$

for all $w \in B V(\Omega) \cap L^{2}(\Omega)$.

Theorem 3.1 Let $\varphi \in L^{1}(\partial \Omega)$. Assume we are under assumptions (H), then the operator $\partial \Phi_{\varphi}$ has dense domain in $L^{2}(\Omega)$ and

$$
\partial \Phi_{\varphi}=\mathcal{B}_{\varphi}
$$

We note that, in the particular case of the nonparametric area integrand $f(x, \xi)=\sqrt{1+\|\xi\|^{2}}$, the characterization of the subdifferential of $\Phi_{\varphi}$ given in Theorem 3.1 coincides with the one given by F. Demengel and R. Temam in [12], Theorem 3.1, where they used a different approach. More precisely, they characterized the subdifferential by means of the duality method of convex optimization introduced by $R$. T. Rockafellar in [19]. To prove Theorem 3.1 we need the following proposition.

Proposition 3.2 Let $\varphi \in L^{1}(\partial \Omega)$. Assume we are under assumptions (H), then $L^{\infty}(\Omega) \subset R\left(I+\mathcal{B}_{\varphi}\right)$ and $D\left(\mathcal{B}_{\varphi}\right)$ is dense in $L^{2}(\Omega)$.

We need to introduce the following sequence of auxiliar operators. For $\varphi \in W^{\frac{1}{2}, 2}(\Omega)$, let

$$
W_{\varphi}^{1,2}(\Omega):=\left\{u \in W^{1,2}(\Omega): u_{\mid \partial \Omega}=\varphi \quad H^{N-1}-\text { a.e. }\right\} .
$$

For every $n \in \mathbb{N}$, consider $\mathbf{a}_{n}(x, \xi):=\mathbf{a}(x, \xi)+\xi / n$. We define the operator $A_{n, \varphi}$ in $L^{2}(\Omega)$ :

$$
(u, v) \in A_{n, \varphi} \Longleftrightarrow u \in W_{\varphi}^{1,2}(\Omega) \cap L^{\infty}(\Omega), v \in L^{2}(\Omega) \text {, and }
$$




$$
\int_{\Omega}(w-u) v d x \leq \int_{\Omega} \mathbf{a}_{n}(x, \nabla u) \cdot \nabla(w-u) d x \quad \forall w \in W_{\varphi}^{1,2}(\Omega) .
$$

A similar proof to the one given in Proposition 1 of [4] give us the following result.

Lemma 3.3 Let $\varphi \in W^{\frac{1}{2}, 2}(\partial \Omega) \cap L^{\infty}(\partial \Omega)$. Then for every $n \in \mathbb{N}$ the operator $A_{n, \varphi}$ satisfies

$$
L^{\infty}(\Omega) \subset R\left(I+A_{n, \varphi}\right) .
$$

We also need an approximation lemma similar to the one given by Anzellotti in [7]. The proof of this lemma will be given in the Appendix.

Lemma 3.4 Let $\Omega$ be an open bounded set in $\mathbb{R}^{N}, N \geq 2$, and assume that $\partial \Omega$ is of class $C^{1}$. If $v, u \in B V(\Omega)$ and $g \in L^{1}(\partial \Omega)$, then there exists a sequence of functions $v_{j} \in C^{1}(\bar{\Omega})$ such that

$(3.8) \int_{\Omega} \sqrt{1+\left|\nabla v_{j}(x)\right|^{2}} d x \rightarrow \int_{\Omega} \sqrt{1+|D v(x)|^{2}} d x+\int_{\partial \Omega}|g-v| d H^{N-1}$,

$$
\begin{gathered}
\nabla v_{j}(x) \rightarrow \nabla v(x) \quad \lambda_{N} \text {-a.e. in } \Omega \\
\left|\nabla v_{j}(x)\right| \rightarrow \infty \text { and } \frac{\nabla v_{j}(x)}{\left|\nabla v_{j}(x)\right|} \rightarrow \frac{D v(x)}{|D v(x)|}|D v|^{s} \text { a.e. in } \Omega, \\
\left|\nabla v_{j}(x)\right| \rightarrow \infty \text { and } \frac{\nabla v_{j}(x)}{\left|\nabla v_{j}(x)\right|} \rightarrow \frac{D u(x)}{|D u(x)|}|D u|^{s s} \text { a.e. in } \Omega,
\end{gathered}
$$

where $|D u|^{s s}$ denotes the part of the singular measure $|D u|^{s}$ which is singular with respect to $|D v|^{s}$,

$$
\begin{gathered}
\left|\nabla v_{j}(x)\right| \rightarrow \infty \text { and } \frac{\nabla v_{j}(x)}{\left|\nabla v_{j}(x)\right|} \rightarrow \frac{g(x)-v(x)}{|g(x)-v(x)|} \nu(x) \\
H^{N-1} \text { a.e. in }\{x \in \Omega: g(x) \neq v(x)\} \\
\left|\nabla v_{j}(x)\right| \rightarrow \infty \text { and } \frac{\nabla v_{j}(x)}{\left|\nabla v_{j}(x)\right|} \rightarrow \frac{v(x)-u(x)}{|v(x)-u(x)|} \nu(x) \\
H^{N-1} \text { a.e. in }\{x \in \Omega: g(x)=v(x), u(x) \neq v(x)\}
\end{gathered}
$$

The next Lemmas will be used to prove Theorem 3.1 and Proposition 3.2. 
Lemma 3.5 Let $\varphi, \varphi_{n} \in L^{1}(\partial \Omega), \varphi_{n} \rightarrow \varphi$ in $L^{1}(\partial \Omega)$. Let $u_{n}, u \in B V(\Omega)$ and $z \in X(\Omega)$ with $\operatorname{div}(z) \in L^{2}(\Omega)$. We assume that

$$
\begin{gathered}
\Phi_{\varphi}\left(u_{n}\right) \rightarrow \Phi_{\varphi}(u), \\
\mathbf{a}\left(x, \nabla u_{n}\right) \rightarrow z \quad \text { weakly } \text { in } L^{\infty}(\Omega), \\
|[z, \nu(x)]| \leq f^{0}(x, \nu(x)) \quad \text { a.e. in } \partial \Omega, \\
\left|z \cdot D^{s} u\right| \leq f^{0}\left(x, D^{s} u\right) \quad \text { as measures in } \Omega, \\
\lim _{n \rightarrow \infty} \int_{\Omega} h\left(x, D u_{n}\right)+\int_{\partial \Omega}\left|u_{n}-\varphi_{n}\right| f^{0}(x, \nu(x)) d H^{N-1}= \\
=\int_{\Omega} h(x, D u)+\int_{\partial \Omega}|u-\varphi| f^{0}(x, \nu(x)) d H^{N-1} . \\
\int_{\Omega} h(x, D u)+\int_{\partial \Omega}|u-\varphi| f^{0}(x, \nu(x)) d H^{N-1} \\
\leq \int_{\Omega}(z, D u)+\int_{\partial \Omega}[z, \nu](\varphi-u) d H^{N-1} .
\end{gathered}
$$

Then

$$
\begin{gathered}
\int_{\Omega} z \cdot \nabla u d x=\int_{\Omega} h(x, \nabla u) d x=\int_{\Omega} \mathbf{a}(x, \nabla u) \cdot \nabla u d x, \\
z \cdot D^{s} u=f^{0}\left(x, D^{s} u\right), \\
{[z, \nu] \in \operatorname{sign}(\varphi-u) f^{0}(x, \nu(x)) \quad H^{N-1}-\text { a.e. }}
\end{gathered}
$$

Proof. By the convexity of $f$, we have

$$
\begin{aligned}
& \int_{\Omega} \mathbf{a}\left(x, \nabla u_{n}\right) \cdot \nabla u d x \leq \\
& \leq \int_{\Omega} \mathbf{a}\left(x, \nabla u_{n}\right) \cdot \nabla u_{n} d x+\int_{\Omega} f(x, \nabla u) d x-\int_{\Omega} f\left(x, \nabla u_{n}\right) d x \\
& \leq \int_{\Omega} \mathbf{a}\left(x, \nabla u_{n}\right) \cdot \nabla u_{n} d x+\int_{\Omega} f^{0}\left(x, D^{s} u_{n}\right)+\int_{\partial \Omega}\left|u_{n}-\varphi_{n}\right| f^{0}(x, \nu(x)) d H^{N-1} \\
& \quad+\int_{\partial \Omega}\left|\varphi_{n}-\varphi\right| f^{0}(x, \nu(x)) d H^{N-1}+\int_{\Omega} f(x, \nabla u) d x \\
& \quad-\left(\int_{\Omega} f\left(x, \nabla u_{n}\right) d x+\int_{\Omega} f^{0}\left(x, D^{s} u_{n}\right)+\int_{\partial \Omega}\left|u_{n}-\varphi\right| f^{0}(x, \nu(x))\right) \\
& =\int_{\Omega} h\left(x, D u_{n}\right) d x+\int_{\partial \Omega}\left|u_{n}-\varphi_{n}\right| f^{0}(x, \nu(x)) d H^{N-1} \\
& \quad+\int_{\partial \Omega}\left|\varphi_{n}-\varphi\right| f^{0}(x, \nu(x)) d H^{N-1}+\int_{\Omega} f(x, \nabla u) d x-\Phi_{\varphi}\left(u_{n}\right) .
\end{aligned}
$$


Letting $n \rightarrow \infty$, and using (3.14), (3.15) and (3.18), we obtain

$$
\begin{aligned}
& \int_{\Omega} z \cdot \nabla u d x \leq \\
& \leq \int_{\Omega} h(x, D u)+\int_{\partial \Omega}|u-\varphi| f^{0}(x, \nu(x)) d H^{N-1}+\int_{\Omega} f(x, \nabla u) d x-\Phi_{\varphi}(u) \\
& =\int_{\Omega} \mathbf{a}(x, \nabla u) \cdot \nabla u d x .
\end{aligned}
$$

Now, since, using (3.16) and (3.17), we have

$$
|[z, \nu](\varphi-u)| \leq|u-\varphi| f^{0}(x, \nu(x))
$$

and

$$
\left|z \cdot D^{s} u\right| \leq f^{0}\left(x, D^{s} u\right)
$$

Hence from (3.19), we obtain (3.20), (3.21) and (3.22).

Lemma 3.6 i) Let $u_{n} \in B V(\Omega) \cap L^{2}(\Omega)$ and $z \in X(\Omega)$. Suppose that

$$
\mathbf{a}\left(x, \nabla u_{n}\right) \rightarrow z \quad \text { weakly* in } L^{\infty}\left(\Omega, \mathbb{R}^{N}\right)
$$

and

$$
\operatorname{div}\left(\mathbf{a}\left(x, \nabla u_{n}\right)\right) \rightarrow \operatorname{div} z \quad \text { weakly in } L^{2}(\Omega) .
$$

Then

$$
\begin{gathered}
{\left[\mathbf{a}\left(x, \nabla u_{n}\right), \nu(x)\right] \rightarrow[z, \nu(x)] \quad \text { weakly in } L^{2}(\partial \Omega) \text { and }} \\
|z(x) \cdot \nu(x)| \leq f^{0}(x, \nu(x)) \quad \text { a.e. in } \partial \Omega .
\end{gathered}
$$

ii) Let $u_{n} \in W^{1,2}(\Omega)$. Let $\mathbf{a}_{n}(x, \xi)=\mathbf{a}(x, \xi)+\xi / n$. Suppose that

$$
\mathbf{a}_{n}\left(x, \nabla u_{n}\right) \rightarrow z \quad \text { weakly in } L^{2}\left(\Omega, \mathbb{R}^{N}\right)
$$

and

$$
\operatorname{div}\left(\mathbf{a}_{n}\left(x, \nabla u_{n}\right)\right) \rightarrow \operatorname{div} z \quad \text { weakly in } L^{2}(\Omega) .
$$

Then

$$
\begin{gathered}
{\left[\mathbf{a}_{n}\left(x, \nabla u_{n}\right), \nu(x)\right] \rightarrow[z, \nu(x)] \quad \text { weakly in } W^{1 / 2,2}(\partial \Omega)^{*} \text { and }} \\
|[z(x), \nu(x)]| \leq f^{0}(x, \nu(x)) \quad \text { a.e. in } \partial \Omega .
\end{gathered}
$$


Proof. Since both proofs are based on similar arguments, we shall only prove $i i)$. Observe that, if $\sigma \in L^{2}\left(\Omega, \mathbb{R}^{N}\right)$ and $\operatorname{div}(\sigma) \in L^{2}(\Omega)$, we can define $[\sigma, \nu]$ using the integration by parts formula

$$
\int_{\partial \Omega}[\sigma, \nu] \psi=\int_{\Omega} \operatorname{div}(\sigma) \psi+\int_{\Omega} \sigma \cdot \nabla \psi .
$$

for all $\psi \in W^{1,2}(\Omega)$. This is consistent with the classical notion of trace at the boundary and it defines $[\sigma, \nu]$ as an element of $W^{1 / 2,2}(\partial \Omega)^{*}$. According to the assumptions (3.29), (3.30) we have that $\left[\mathbf{a}_{n}\left(x, \nabla u_{n}\right), \nu(x)\right] \rightarrow[z, \nu(x)]$ weakly in $W^{1 / 2,2}(\partial \Omega)^{*}$. In $i$ ), the analogous conclusion (3.25) follows from the results in [5] and the fact that $\mathbf{a}\left(x, \nabla u_{n}\right)$ is uniformly bounded in $L^{\infty}(\Omega)$. In this case, the traces $\left[\mathbf{a}\left(x, \nabla u_{n}\right), \nu(x)\right]$ are in $L^{\infty}(\partial \Omega)$.

To prove (3.32), again, we observe that [16] if $\sigma \in L^{2}\left(\Omega, \mathbb{R}^{N}\right)$ and $\operatorname{div}(\sigma) \in L^{2}(\Omega)$, then there is a sequence $\sigma_{k} \in C^{\infty}\left(\bar{\Omega}, \mathbb{R}^{N}\right)$ satisfying

$$
\begin{gathered}
\sigma_{k} \rightarrow \sigma \quad \text { in } L^{2}\left(\Omega, \mathbb{R}^{N}\right), \\
\operatorname{div} \sigma_{k} \rightarrow \operatorname{div} \sigma \quad \text { in } L^{2}(\Omega) .
\end{gathered}
$$

We recall the construction in [16]. We use a partition of unity $\theta_{j}, j=$ $1,2, \ldots p$, in $\bar{\Omega}$ with $0 \leq \theta_{j} \leq 1, \theta_{j} \in C_{0}^{\infty}\left(\mathbb{R}^{N}\right)$, such that if the support of $\theta_{j}$ intersects $\partial \Omega$, then for some bounded open cone $K_{j}$ with vertex 0 , every $x \in \partial \Omega \cap \operatorname{supp}\left(\theta_{j}\right)$ satisfies $\left(x+K_{j}\right) \cap \bar{\Omega}=\emptyset$, and for some $r>0$, every $x \in \partial \Omega \cap\left(\operatorname{supp}\left(\theta_{j}\right)+B(0, r)\right)$ satisfies $\left(x-K_{j}\right) \subset \Omega$. For each $j$, we choose $\rho_{j} \in C_{0}^{\infty}\left(\mathbb{R}^{N}\right), 0 \leq \rho_{j} \leq 1$, with $\int_{\mathbb{R}^{N}} \rho_{j} d x=1$, and let $\rho_{j, k}(x)=k^{N} \rho_{j}(k x)$. If $j$ is such that the support of $\theta_{j}$ intersects $\partial \Omega$, we choose $\rho_{j}$ such that $\operatorname{supp}\left(\rho_{j}\right) \subseteq K_{j}$. Then we define

$$
\sigma_{k}=\sum_{j=1}^{p} \rho_{j, k} *\left(\theta_{j} \sigma \chi_{\Omega}\right)
$$

As it was proved in [16], $\sigma_{k}$ satisfies (3.34) and (3.35). As in the first part of the proof, we have that

$$
\int_{\partial \Omega}\left[\sigma_{k}, \nu\right] \psi \rightarrow \int_{\partial \Omega}[\sigma, \nu] \psi
$$

for all $\psi \in W^{1,2}(\Omega)$. We shall use this observation for $\sigma=\mathbf{a}_{n}\left(x, \nabla u_{n}\right)$. Previously, we extend $u_{n}$ as a function in $W^{1,2}\left(\mathbb{R}^{N}\right)$ such that $\left\|u_{n}\right\|_{W^{1,2}\left(\mathbb{R}^{N}\right)} \leq$ $C\left\|u_{n}\right\|_{W^{1,2}(\Omega)}$ for some constant $C>0$ depending only on $\Omega([1])$. Then we define

$$
\mathbf{a}_{n, k}\left(x, \nabla u_{n}\right)=\sum_{j=1}^{p} \rho_{j, k} *\left(\theta_{j} \mathbf{a}\left(x, \nabla u_{n}\right) \chi_{\Omega}+\theta_{j} \frac{\nabla u_{n}}{n}\right) .
$$


Now, since $\mathbf{a}_{n, k}\left(x, \nabla u_{n}\right) \in C^{\infty}(\bar{\Omega}),\left[\mathbf{a}_{n, k}\left(x, \nabla u_{n}\right), \nu(x)\right]$ can be understood in a classical sense. Let $\psi \in W^{1 / 2,2}(\partial \Omega)$. We may write

$$
\begin{aligned}
\int_{\partial \Omega}\left[\mathbf{a}_{n, k}\left(x, \nabla u_{n}\right), \nu(x)\right] \psi & =\sum_{j=1}^{p} \int_{\partial \Omega}\left[\rho_{j, k} *\left(\theta_{j} \mathbf{a}\left(x, \nabla u_{n}\right) \chi_{\Omega}\right), \nu(x)\right] \psi(x)+ \\
+ & \sum_{j=1}^{p} \frac{1}{n} \int_{\partial \Omega}\left[\rho_{j, k} *\left(\theta_{j} \nabla u_{n}\right), \nu(x)\right] \psi(x) .
\end{aligned}
$$

By taking $k$ sufficiently large, we may assume that all $\theta_{j}$ used in the above expression are such that $\operatorname{supp}\left(\theta_{j}\right)$ intersects $\partial \Omega$. We observe that

$$
\begin{aligned}
& \int_{\partial \Omega}\left|\left[\rho_{j, k} *\left(\theta_{j} \mathbf{a}\left(x, \nabla u_{n}\right) \chi_{\Omega}\right), \nu(x)\right]\right||\psi(x)| \leq \\
& \leq \int_{\partial \Omega} \int_{\Omega} \rho_{j, k}(x-y) \theta_{j}(y)\left|\mathbf{a}\left(y, \nabla u_{n}(y)\right) \cdot \nu(x)\right||\psi(x)| d y d x \\
& \leq \int_{\partial \Omega} \int_{\Omega} \rho_{j, k}(x-y) \theta_{j}(y) f^{0}(y, \nu(x))|\psi(x)| d y d x .
\end{aligned}
$$

Since

$$
\nabla u_{n} \theta_{j}=\nabla\left(u_{n} \theta_{j}\right)-u_{n} \nabla \theta_{j}
$$

we may write

$$
\begin{aligned}
\int_{\partial \Omega}\left[\rho_{j, k} *\left(\theta_{j} \nabla u_{n}\right), \nu(x)\right] \psi(x)= \\
\quad=\int_{\partial \Omega}\left[\nabla \rho_{j, k} *\left(\theta_{j} u_{n}\right), \nu(x)\right] \psi(x)-\int_{\partial \Omega}\left[\rho_{j, k} *\left(\nabla \theta_{j} u_{n}\right), \nu(x)\right] \psi(x) .
\end{aligned}
$$

We estimate both integrals in the right hand side of the above expression. First,

$$
\begin{aligned}
\left|\int_{\partial \Omega}\left[\nabla \rho_{j, k} *\left(\theta_{j} u_{n}\right), \nu(x)\right] \psi(x)\right| \leq \\
\quad \leq\left\|\frac{\partial}{\partial \nu}\left(\rho_{j, k} *\left(u_{n} \theta_{j}\right)\right)\right\|_{W^{1 / 2,2}(\partial \Omega)^{*}}\|\psi\|_{W^{1 / 2,2}(\partial \Omega)} \\
\quad \leq C\left\|\rho_{j, k} *\left(u_{n} \theta_{j}\right)\right\|_{W^{1,2}(\Omega)}\|\psi\|_{W^{1 / 2,2}(\partial \Omega)} \\
\leq C\left\|\rho_{j, k} *\left(u_{n} \theta_{j}\right)\right\|_{W^{1,2}\left(\mathbb{R}^{N}\right)}\|\psi\|_{W^{1 / 2,2}(\partial \Omega)} \\
\leq C\left\|u_{n} \theta_{j}\right\|_{W^{1,2}\left(\mathbb{R}^{N}\right)}\|\psi\|_{W^{1 / 2,2}(\partial \Omega)} \leq C\left\|u_{n}\right\|_{W^{1,2}\left(\mathbb{R}^{N}\right)}\|\psi\|_{W^{1 / 2,2}(\partial \Omega)} \\
\leq C\left\|u_{n}\right\|_{W^{1,2}(\Omega)}\|\psi\|_{W^{1,2}(\Omega)}
\end{aligned}
$$


for some constant $C>0$ (which may change from line to line). A similar analysis proves that

$$
\left|\int_{\partial \Omega}\left[\rho_{j, k} *\left(\nabla \theta_{j} u_{n}\right), \nu(x)\right] \psi(x)\right| \leq C\left\|u_{n}\right\|_{W^{1,2}(\Omega)}\|\psi\|_{W^{1,2}(\Omega)}
$$

for some constant $C>0$. Taking all the above into account, we obtain

$$
\begin{aligned}
& \left|\int_{\partial \Omega}\left[\mathbf{a}_{n, k}\left(x, \nabla u_{n}\right), \nu(x)\right] \psi\right| \leq \\
& \quad \leq \sum_{j=1}^{p} \int_{\partial \Omega} \int_{\Omega} \rho_{j, k}(x-y) \theta_{j}(y) f^{0}(y, \nu(x))|\psi(x)| d y d x \\
& \quad+\frac{C}{n}\left\|u_{n}\right\|_{W^{1,2}(\Omega)}\|\psi\|_{W^{1,2}(\Omega)} .
\end{aligned}
$$

Letting $k \rightarrow \infty$, and taking into account the fact that $\theta_{j}$ is a partition of unity in $\bar{\Omega}$ and our assumptions on $\theta_{j}$ and $K_{j}$, we obtain

$\left|\int_{\partial \Omega}\left[\mathbf{a}_{n}\left(x, \nabla u_{n}\right), \nu(x)\right] \psi\right| \leq \int_{\partial \Omega} f^{0}(x, \nu(x))|\psi(x)| d x+\frac{C}{n}\left\|u_{n}\right\|_{W^{1,2}(\Omega)}\|\psi\|_{W^{1,2}(\Omega)}$

Now, letting $n \rightarrow \infty$, and using (3.27), (3.28), we obtain

$$
\left|\int_{\partial \Omega}[z, \nu(x)] \psi\right| \leq \int_{\partial \Omega} f^{0}(x, \nu(x))|\psi(x)| d x
$$

for all $\psi \in W^{1,2}(\Omega)$. Now, since $z \in L^{\infty}(\Omega)$ and $\operatorname{div}(z) \in L^{2}(\Omega),[z, \nu]$ coincides with the trace given in the sense of Anzellotti $([5])$, and, therefore, $[z, \nu] \in L^{\infty}(\partial \Omega)$. Hence, from (3.36), we conclude that $|[z(x), \nu(x)]| \leq$ $f^{0}(x, \nu(x))$.

Lemma 3.7 Suppose that any of the assumptions of Lemma 3.6 hold. Moreover we assume that

$$
\begin{aligned}
& u_{n} \rightarrow u \quad \text { in } L^{2}(\Omega), \quad\left\|u_{n}\right\|_{B V} \quad \text { is bounded } \\
& \text { and } \mathbf{a}\left(x, \nabla u_{n}\right) \cdot D^{s} u_{n}=f^{0}\left(x, D^{s} u_{n}\right)
\end{aligned}
$$

Then

$$
z(x)=\mathbf{a}(x, \nabla u(x)) \quad \text { a.e. } x \in \Omega .
$$


Proof. Again, since both proofs are based on similar arguments, we shall only prove (3.38) under the assumptions given in $i$ ) of Lemma 3.6. Let $0 \leq \phi \in C_{0}^{1}(\Omega)$ and $g \in C^{1}(\bar{\Omega})$. We observe that

$$
\begin{aligned}
\int_{\Omega} \phi\left[\left(\mathbf{a}\left(x, \nabla u_{n}\right), D\left(u_{n}-g\right)\right)\right. & \left.-\mathbf{a}(x, \nabla g) D\left(u_{n}-g\right)\right]= \\
= & \left.\int_{\Omega} \phi\left[\mathbf{a}\left(x, \nabla u_{n}\right)-\mathbf{a}(x, \nabla g)\right) \cdot \nabla\left(u_{n}-g\right)\right] d x \\
& \left.+\int_{\Omega} \phi\left[\mathbf{a}\left(x, \nabla u_{n}\right)-\mathbf{a}(x, \nabla g)\right] \cdot D^{s}\left(u_{n}-g\right)\right) .
\end{aligned}
$$

Since both terms at the right hand side of the above expression are positive, we have

$$
\int_{\Omega} \phi\left[\left(\mathbf{a}\left(x, \nabla u_{n}\right), D\left(u_{n}-g\right)\right)-\mathbf{a}(x, \nabla g) D\left(u_{n}-g\right)\right] \geq 0 .
$$

Since

$$
\begin{aligned}
& \int_{\Omega} \phi\left(\mathbf{a}\left(x, \nabla u_{n}\right), D\left(u_{n}-g\right)\right)= \\
& \quad=-\int_{\Omega} \operatorname{div}\left(\mathbf{a}\left(x, \nabla u_{n}\right)\right) \phi\left(u_{n}-g\right) d x-\int_{\Omega}\left(u_{n}-g\right) \mathbf{a}\left(x, \nabla u_{n}\right) \cdot \nabla \phi d x
\end{aligned}
$$

we get

$$
\begin{aligned}
& \lim _{n \rightarrow \infty} \int_{\Omega} \phi\left(\mathbf{a}\left(x, \nabla u_{n}\right), D\left(u_{n}-g\right)\right)= \\
& =-\int_{\Omega} \operatorname{div}(z) \phi(u-g) d x-\int_{\Omega}(u-g) z \cdot \nabla \phi d x=\int_{\Omega} \phi(z, D(u-g)) .
\end{aligned}
$$

On the other hand,

$$
\lim _{n \rightarrow \infty} \int_{\Omega} \phi \mathbf{a}(x, \nabla g) D\left(u_{n}-g\right)=\int_{\Omega} \phi \mathbf{a}(x, \nabla g) D(u-g) .
$$

Consequently, we obtain

$$
\int_{\Omega} \phi[(z, D(u-g))-\mathbf{a}(x, \nabla g) D(u-g)] \geq 0, \quad \forall 0 \leq \phi \in C_{0}^{1}(\Omega) .
$$

Thus the measure $(z, D(u-g))-\mathbf{a}(x, \nabla g) D(u-g) \geq 0$. Then its absolutely continuous part

$$
(z-\mathbf{a}(x, \nabla g)) \cdot \nabla(u-g) \geq 0 \quad \text { a.e. in } \Omega .
$$


Since we may take a countable set dense in $C^{1}(\bar{\Omega})$ we have that the above inequality holds for all $x \in \tilde{\Omega}$, where $\tilde{\Omega} \subset \Omega$ is such that $\lambda_{N}(\Omega \backslash \tilde{\Omega})=0$, and all $g \in C^{1}(\bar{\Omega})$. Now, fixed $x \in \tilde{\Omega}$ and given $\xi \in \mathbb{R}^{N}$, there is $g \in C^{1}(\bar{\Omega})$ such that $\nabla g(x)=\xi$. Then

$$
(z(x)-\mathbf{a}(x, \xi)) \cdot(\nabla u(x)-\xi) \geq 0, \quad \forall \xi \in R^{N} .
$$

These inequalities imply (3.38) by an application of Minty-Browder's method in $\mathbb{R}^{N}$.

Proof of Proposition 3.2 We divide the proof in three steps.

Step 1. Suppose first that $\varphi \in C^{1}(\bar{\Omega})$. Let $v \in L^{\infty}(\Omega)$. We shall find $u \in$ $B V(\Omega) \cap L^{2}(\Omega)$ such that $(u, v-u) \in \mathcal{B}_{\varphi}$. That is, there is $\mathbf{a}(x, \nabla u) \in X(\Omega)$ satisfying

$$
\begin{gathered}
(v-u)=-\operatorname{div} \mathbf{a}(x, \nabla u), \quad \text { in } \mathcal{D}^{\prime}(\Omega), \\
\mathbf{a}(x, \nabla u) \cdot D^{s} u=f^{0}\left(x, D^{s} u\right), \quad \text { and } \\
{[\mathbf{a}(x, \nabla u), \nu] \in \operatorname{sign}(\varphi-u) f^{0}(x, \nu(x)) \quad H^{N-1}-\text { a.e. }}
\end{gathered}
$$

By Lemma 3.3, we know that for any $n \in \mathbb{N}$ there exists $u_{n} \in W_{\varphi}^{1,2}(\Omega) \cap$ $L^{\infty}(\Omega)$ such that $\left(u_{n}, v-u_{n}\right) \in A_{n, \varphi}$. Hence

$$
\int_{\Omega}\left(w-u_{n}\right)\left(v-u_{n}\right) d x \leq \int_{\Omega} \mathbf{a}_{n}\left(x, \nabla u_{n}\right) \cdot \nabla\left(w-u_{n}\right) d x
$$

for all $w \in W_{\varphi}^{1,2}(\Omega)$.

Let $M_{1}:=\sup \left\{\|\varphi\|_{\infty},\|v\|_{\infty}\right\}$. Then, taking $w=u_{n}-\left(u_{n}-M_{1}\right)^{+}$as test function in (3.42), we obtain

$$
\int_{\Omega}\left(u_{n}-M_{1}\right)^{+}\left(u_{n}-v\right) d x \leq 0 .
$$

Hence,

$$
\begin{aligned}
\int_{\left\{u_{n}>M_{1}\right\}}\left(u_{n}-M_{1}\right)^{2} d x & \leq \int_{\left\{u_{n}>M_{1}\right\}}\left(u_{n}-M_{1}\right)\left(u_{n}-v\right) d x \\
& =\int_{\Omega}\left(u_{n}-M_{1}\right)^{+}\left(u_{n}-v\right) d x \leq 0 .
\end{aligned}
$$

Consequently, $u_{n} \leq M_{1}$ a.e. in $\Omega$. Analogously, taking $w=u_{n}+\left(u_{n}+M_{1}\right)^{-}$ as test function, we get $-M_{1} \leq u_{n}$ a.e. in $\Omega$. Therefore,

$$
\left\|u_{n}\right\|_{\infty} \leq M_{1} \quad \text { for all } n \in \mathbb{N} \text {. }
$$


Taking $w=w_{0} \in W_{\varphi}^{1,2}(\Omega) \cap L^{\infty}(\Omega)$ in (3.42), applying Young's inequality and using (3.43) we get

$$
\begin{aligned}
& \int_{\Omega} \mathbf{a}\left(x, \nabla u_{n}\right) \cdot \nabla u_{n} d x+\frac{1}{n} \int_{\Omega}\left|\nabla u_{n}\right|^{2} d x \leq \\
& \leq \int_{\Omega} \mathbf{a}\left(x, \nabla u_{n}\right) \cdot \nabla w_{0} d x+\frac{1}{n} \int_{\Omega} \nabla u_{n} \cdot \nabla w_{0} d x+\int_{\Omega}\left(w_{0}-u_{n}\right)\left(u_{n}-v\right) d x \\
& \leq M_{2}\left(\int_{\Omega}\left|\nabla w_{0}\right|^{2} d x\right)^{\frac{1}{2}}+\frac{1}{2 n} \int_{\Omega}\left|\nabla u_{n}\right|^{2} d x+\frac{1}{2 n} \int_{\Omega}\left|\nabla w_{0}\right|^{2} d x+M_{3} \\
& \leq M_{4}+\frac{1}{2 n} \int_{\Omega}\left|\nabla u_{n}\right|^{2} d x .
\end{aligned}
$$

Hence, by (2.5), we obtain

$$
\int_{\Omega}\left|\nabla u_{n}\right| d x \leq M_{5} \quad \forall n \in \mathbb{N}
$$

and

$$
\frac{1}{n} \int_{\Omega}\left|\nabla u_{n}\right|^{2} d x \leq M_{6} \quad \forall n \in \mathbb{N}
$$

Thus, $\left\{u_{n}: n \in \mathbb{N}\right\}$ is bounded in $W^{1,1}(\Omega)$ and, by extracting a subsequence if is necessary, we may assume that $u_{n}$ converges in $L^{1}(\Omega)$ and converges almost everywhere to some $u \in L^{1}(\Omega)$ as $n \rightarrow+\infty$. Now, by (3.43) and (3.44), we have that $u_{n} \rightarrow u$ in $L^{2}(\Omega)$ and $u \in B V(\Omega) \cap L^{\infty}(\Omega)$.

Observe that by (2.4) and (3.45), $\left\{\mathbf{a}_{n}\left(x, \nabla u_{n}\right): n \in \mathbb{N}\right\}$ is bounded in $L^{2}\left(\Omega, \mathbb{R}^{N}\right)$. Consequently we may assume that

$$
\mathbf{a}_{n}\left(x, \nabla u_{n}\right) \rightarrow z \quad \text { as } n \rightarrow \infty \text {, weakly in } L^{2}\left(\Omega, \mathbb{R}^{N}\right) .
$$

Given $\psi \in C_{0}^{\infty}(\Omega)$, taking $w=u_{n} \pm \psi$ in (3.42) we obtain

$$
\int_{\Omega} \psi\left(v-u_{n}\right) d x=\int_{\Omega} \mathbf{a}_{n}\left(x, \nabla u_{n}\right) \cdot \nabla \psi d x .
$$

Letting $n \rightarrow+\infty$, we obtain

$$
\int_{\Omega}(v-u) \psi d x=\int_{\Omega} z \cdot \nabla \psi d x,
$$

that is,

$$
v-u=-\operatorname{div}(z), \quad \text { in } \mathcal{D}^{\prime}(\Omega)
$$


and

$$
\operatorname{div} \mathbf{a}_{n}\left(x, \nabla u_{n}\right) \rightarrow \operatorname{div}(z) \quad \text { weakly in } L^{2}(\Omega) .
$$

Since, by (3.45),

$$
\frac{1}{n}\left|\nabla u_{n}\right| \rightarrow 0 \quad \text { in } \quad L^{2}(\Omega),
$$

as a consequence of (3.46), it follows that

$$
\mathbf{a}\left(x, \nabla u_{n}\right) \rightarrow z \quad \text { as } n \rightarrow \infty, \text { weakly in } L^{2}\left(\Omega, \mathbb{R}^{N}\right) .
$$

Moreover, by (2.4) we may assume that

$$
\mathbf{a}\left(x, \nabla u_{n}\right) \rightarrow z \quad \text { as } n \rightarrow \infty \text {, weakly* in } L^{\infty}\left(\Omega, \mathbb{R}^{N}\right) .
$$

Let us prove that

$$
\lim _{n \rightarrow \infty} \int_{\Omega} \mathbf{a}\left(x, \nabla u_{n}\right) \cdot \nabla u_{n} d x=\int_{\Omega}(z, D u)-\int_{\partial \Omega}[z, \nu](u-\varphi) d H^{N-1}
$$

By (3.42), we have

$$
\begin{aligned}
& \int_{\Omega}\left(w-u_{n}\right)\left(v-u_{n}\right) d x+\int_{\Omega} \mathbf{a}\left(x, \nabla u_{n}\right) \cdot \nabla u_{n} d x \leq \\
& \leq \int_{\Omega} \mathbf{a}\left(x, \nabla u_{n}\right) \cdot \nabla w d x+\frac{1}{n} \int_{\Omega} \nabla u_{n} \cdot \nabla w d x
\end{aligned}
$$

for all $w \in W_{\varphi}^{1,2}(\Omega)$. By Lemma 3.4, there exists $v_{j} \in C^{1}(\bar{\Omega})$ such that $v_{j \mid \partial \Omega}=\varphi, v_{j} \rightarrow u$ in $L^{1}(\Omega)$. If we set $w=v_{j}$ in (3.53), taking the upper limit when $n \rightarrow \infty$, we get

$$
\int_{\Omega}\left(v_{j}-u\right)(v-u) d x+\limsup _{n \rightarrow \infty} \int_{\Omega} \mathbf{a}\left(x, \nabla u_{n}\right) \cdot \nabla u_{n} d x \leq \int_{\Omega} z \cdot \nabla v_{j} d x .
$$

Now, by Green's formula we have

$$
\begin{aligned}
\int_{\Omega} z \cdot \nabla v_{j} d x & =-\int_{\Omega} \operatorname{div}(z) v_{j} d x+\int_{\partial \Omega}[z, \nu] \varphi d H^{N-1} \\
& =\int_{\Omega}(v-u) v_{j} d x+\int_{\partial \Omega}[z, \nu] \varphi d H^{N-1}
\end{aligned}
$$

Hence, taking limit as $j \rightarrow \infty$ and applying again the Green's formula we obtain that

$$
\lim _{j \rightarrow \infty} \int_{\Omega} z \cdot \nabla v_{j} d x=\int_{\Omega}(z, D u)-\int_{\partial \Omega}[z, \nu](u-\varphi) d H^{N-1} .
$$


Letting $j \rightarrow \infty$ in (3.54), we have

(3.56) $\limsup _{n \rightarrow \infty} \int_{\Omega} \mathbf{a}\left(x, \nabla u_{n}\right) \cdot \nabla u_{n} d x \leq \int_{\Omega}(z, D u)-\int_{\partial \Omega}[z, \nu](u-\varphi) d H^{N-1}$.

On the other hand,

$$
\begin{gathered}
\int_{\Omega} \mathbf{a}\left(x, \nabla u_{n}\right) \cdot \nabla u_{n} d x=\int_{\Omega}\left(\mathbf{a}\left(x, \nabla u_{n}\right)-\mathbf{a}\left(x, \nabla v_{j}\right)\right) \cdot \nabla\left(u_{n}-v_{j}\right) d x+ \\
\quad+\int_{\Omega}\left(\mathbf{a}\left(x, \nabla u_{n}\right)-\mathbf{a}\left(x, \nabla v_{j}\right)\right) \cdot \nabla v_{j} d x+\int_{\Omega} \mathbf{a}\left(x, \nabla v_{j}\right) \cdot \nabla u_{n} d x \geq \\
\quad \geq \int_{\Omega}\left(\mathbf{a}\left(x, \nabla u_{n}\right)-\mathbf{a}\left(x, \nabla v_{j}\right)\right) \cdot \nabla v_{j} d x+\int_{\Omega} \mathbf{a}\left(x, \nabla v_{j}\right) \cdot \nabla u_{n} d x .
\end{gathered}
$$

Hence

$$
\begin{aligned}
\liminf _{n \rightarrow \infty} \int_{\Omega} \mathbf{a}\left(x, \nabla u_{n}\right) & \cdot \nabla u_{n} d x \geq \lim _{n \rightarrow \infty}\left(\int_{\Omega} \mathbf{a}\left(x, \nabla u_{n}\right) \cdot \nabla v_{j} d x\right. \\
& \left.-\int_{\Omega} \mathbf{a}\left(x, \nabla v_{j}\right) \cdot \nabla v_{j} d x+\int_{\Omega} \mathbf{a}\left(x, \nabla v_{j}\right) \cdot \nabla u_{n} d x\right) .
\end{aligned}
$$

If we consider the $\mathbb{R}^{N}$-valued measures $\mu_{n}, \mu$ on $\bar{\Omega}$ which are defined as

$$
\begin{aligned}
\mu_{n}(B) & :=\int_{B \cap \Omega} \nabla u_{n} d x \\
\mu(B) & :=\int_{B \cap \Omega} D u+\int_{B \cap \partial \Omega}(\varphi-u) \nu d H^{N-1}
\end{aligned}
$$

for all Borel sets $B \subset \bar{\Omega}$, we have

$$
\mu_{n} \rightarrow \mu \quad \text { weakly as measures in } \bar{\Omega} \text {. }
$$

Then, since $\mathbf{a}\left(x, \nabla v_{j}(x)\right) \in C\left(\bar{\Omega}, \mathbb{R}^{N}\right)$, we have

$$
\begin{aligned}
\lim _{n \rightarrow \infty} \int_{\Omega} \mathbf{a}\left(x, \nabla v_{j}\right) \cdot \nabla u_{n} d x= \\
\quad=\int_{\Omega} \mathbf{a}\left(x, \nabla v_{j}\right) d D u+\int_{\partial \Omega} \mathbf{a}\left(x, \nabla v_{j}\right) \cdot \nu(\varphi-u) d H^{N-1} .
\end{aligned}
$$

Therefore, we have

$$
\begin{gathered}
\liminf _{n \rightarrow \infty} \int_{\Omega} \mathbf{a}\left(x, \nabla u_{n}\right) \cdot \nabla u_{n} d x \geq \int_{\Omega} z \cdot \nabla v_{j} d x-\int_{\Omega} \mathbf{a}\left(x, \nabla v_{j}\right) \cdot \nabla v_{j} d x \\
+\int_{\Omega} \mathbf{a}\left(x, \nabla v_{j}\right) d D u+\int_{\partial \Omega} \mathbf{a}\left(x, \nabla v_{j}\right) \cdot \nu(\varphi-u) d H^{N-1} .
\end{gathered}
$$


Now, by Theorem 7.4 of [7], we have

$$
\begin{aligned}
\lim _{j \rightarrow \infty} & \int_{\Omega} \mathbf{a}\left(x, \nabla v_{j}\right) \cdot \nabla v_{j} d x= \\
= & \int_{\Omega} \mathbf{a}(x, \nabla u) \cdot \nabla u d x+\int_{\Omega} \mathbf{a}^{\infty}\left(x, \overrightarrow{D^{s} u}\right) \cdot D^{s} u \\
& \quad+\int_{\partial \Omega} \mathbf{a}^{\infty}(x,(\varphi-u) \nu) \cdot \nu(\varphi-u) d H^{N-1} .
\end{aligned}
$$

On the other hand, as a consequence of Lemma 3.4, we have

$$
\begin{aligned}
& \lim _{j \rightarrow \infty} \int_{\Omega} \mathbf{a}\left(x, \nabla v_{j}\right) d D u= \\
& \quad=\lim _{j \rightarrow \infty}\left(\int_{\Omega} \mathbf{a}\left(x, \nabla v_{j}\right) \cdot \nabla u d x+\int_{\Omega} \mathbf{a}\left(x, \nabla v_{j}\right) d D^{s} u\right) \\
& \quad=\int_{\Omega} \mathbf{a}(x, \nabla u) \cdot \nabla u d x+\int_{\Omega} \mathbf{a}^{\infty}\left(x, \overrightarrow{D^{s} u}\right) \cdot D^{s} u
\end{aligned}
$$

and

$$
\begin{aligned}
& \lim _{j \rightarrow \infty} \int_{\partial \Omega} \mathbf{a}\left(x, \nabla v_{j}\right) \cdot \nu(\varphi-u) d H^{N-1} \\
& \quad=\int_{\partial \Omega} \mathbf{a}^{\infty}\left(x, \frac{\varphi-u}{|\varphi-u|} \nu\right) \cdot \nu(\varphi-u) d H^{N-1}= \\
& \quad=\int_{\partial \Omega} \mathbf{a}^{\infty}(x,(\varphi-u) \nu) \cdot \nu(\varphi-u) d H^{N-1} .
\end{aligned}
$$

Collecting all these facts, we obtain

$$
\begin{aligned}
\liminf _{n \rightarrow \infty} \int_{\Omega} \mathbf{a}\left(x, \nabla u_{n}\right) \cdot \nabla u_{n} d x & \geq \lim _{j \rightarrow \infty} \int_{\Omega} z \cdot \nabla v_{j} d x \\
& =\int_{\Omega}(z, D u)-\int_{\partial \Omega}[z, \nu](u-\varphi) d H^{N-1} .
\end{aligned}
$$

Combining this inequality with (3.56), we obtain (3.52).

Our next purpose will be to show that

$$
\begin{aligned}
\int_{\Omega} h(x, D u) & +\int_{\partial \Omega}|\varphi-u| f^{0}(x, \nu(x)) d H^{N-1} \\
& =\int_{\Omega}(z, D u)-\int_{\partial \Omega}[z, \nu](u-\varphi) d H^{N-1} .
\end{aligned}
$$

According to [6], there exists a sequence $\left\{w_{j}\right\} \subset C^{1}(\Omega) \cap B V(\Omega)$ such that $w_{j \mid \partial \Omega}=\varphi$,

$$
w_{j} \rightarrow u \text { in } L^{1}(\Omega), \quad \text { and } \quad \Phi_{\varphi}\left(w_{j}\right) \rightarrow \Phi_{\varphi}(u)
$$


Now, by the convexity of $f$, we have

$$
\begin{aligned}
\int_{\Omega} f\left(x, \nabla u_{n}\right) d x \leq & \int_{\Omega} \mathbf{a}\left(x, \nabla u_{n}\right) \cdot \nabla u_{n} d x \\
& -\int_{\Omega} \mathbf{a}\left(x, \nabla u_{n}\right) \cdot \nabla w_{j} d x+\int_{\Omega} f\left(x, \nabla w_{j}\right) d x .
\end{aligned}
$$

Thus,

$$
\Phi_{\varphi}\left(u_{n}\right) \leq \int_{\Omega} \mathbf{a}\left(x, \nabla u_{n}\right) \cdot \nabla u_{n} d x-\int_{\Omega} \mathbf{a}\left(x, \nabla u_{n}\right) \cdot \nabla w_{j} d x+\Phi_{\varphi}\left(w_{j}\right) .
$$

Using (3.52), it follows that

$$
\begin{gathered}
\limsup _{n \rightarrow \infty} \Phi_{\varphi}\left(u_{n}\right) \leq \\
\leq \int_{\Omega}(z, D u)-\int_{\partial \Omega}[z, \nu](u-\varphi) d H^{N-1}-\lim _{n \rightarrow \infty} \int_{\Omega} \mathbf{a}\left(x, \nabla u_{n}\right) \cdot \nabla w_{j} d x+\Phi_{\varphi}\left(w_{j}\right) \\
=\int_{\Omega}(z, D u)-\int_{\partial \Omega}[z, \nu](u-\varphi) d H^{N-1}-\int_{\Omega} z \cdot \nabla w_{j} d x+\Phi_{\varphi}\left(w_{j}\right) .
\end{gathered}
$$

Since

$$
\begin{aligned}
& \lim _{j \rightarrow \infty} \int_{\Omega} z \cdot \nabla w_{j} d x=\lim _{j \rightarrow \infty}\left(-\int_{\Omega} \operatorname{div}(z) w_{j} d x+\int_{\partial \Omega}[z, \nu] \varphi d H^{N-1}\right)= \\
= & -\int_{\Omega} \operatorname{div}(z) u d x+\int_{\partial \Omega}[z, \nu] \varphi d H^{N-1}=\int_{\Omega}(z, D u)-\int_{\partial \Omega}[z, \nu](u-\varphi) d H^{N-1},
\end{aligned}
$$

letting $j \rightarrow \infty$ in the above inequality, we obtain

$$
\limsup _{n \rightarrow \infty} \Phi_{\varphi}\left(u_{n}\right) \leq \lim _{j \rightarrow \infty} \Phi_{\varphi}\left(w_{j}\right)=\Phi_{\varphi}(u)
$$

Thus, by the lower-semicontinuity of $\Phi_{\varphi}$, we get

$$
\Phi_{\varphi}(u)=\lim _{n \rightarrow \infty} \Phi_{\varphi}\left(u_{n}\right)
$$

Now,

$$
\Phi_{\varphi}(u)=\int_{\bar{\Omega}} \tilde{f}(x, \tilde{\mu}) \quad \text { and } \quad \Phi_{\varphi}\left(u_{n}\right)=\int_{\bar{\Omega}} \tilde{f}\left(x, \tilde{\mu}_{n}\right) .
$$

Hence, (3.58) yields

$$
\lim _{n \rightarrow \infty} \int_{\bar{\Omega}} \tilde{f}\left(x, \tilde{\mu}_{n}\right)=\int_{\bar{\Omega}} \tilde{f}(x, \tilde{\mu})
$$


Then, applying Theorem 3 of [18], it follows that

$$
\int_{\bar{\Omega}} \tilde{h}(x, \tilde{\mu})=\lim _{n \rightarrow \infty} \int_{\bar{\Omega}} \tilde{h}\left(x, \tilde{\mu}_{n}\right)=\lim _{n \rightarrow \infty} \int_{\Omega} \mathbf{a}\left(x, \nabla u_{n}\right) \cdot \nabla u_{n} .
$$

Since

$$
\begin{aligned}
\int_{\bar{\Omega}} \tilde{h}(x, \tilde{\mu})= & \int_{\bar{\Omega}} \tilde{h}\left(x, \tilde{\mu}^{a}(x)\right) d x+\int_{\bar{\Omega}} \tilde{h}\left(x, \frac{d \tilde{\mu}^{s}}{d\left|\tilde{\mu}^{s}\right|}(x)\right) d\left|\tilde{\mu}^{s}\right| \\
= & \int_{\bar{\Omega}} \tilde{h}\left(x, \mu^{a}(x), 1\right) d x+\int_{\bar{\Omega}} \tilde{h}\left(x, \frac{d \mu^{s}}{d\left|\mu^{s}\right|}(x), 0\right) d\left|\mu^{s}\right| \\
= & \int_{\bar{\Omega}} h\left(x, \mu^{a}(x)\right) d x+\int_{\bar{\Omega}} h^{0}\left(x, \frac{d \mu^{s}}{d\left|\mu^{s}\right|}(x)\right) d\left|\mu^{s}\right| \\
= & \int_{\Omega} h(x, \nabla u(x)) d x+\int_{\Omega} h^{0}\left(x, \overrightarrow{D^{s} u}(x)\right) d\left|D^{s} u\right| \\
& +\int_{\partial \Omega} h^{0}\left(x, \frac{(\varphi-u) \cdot \nu}{|(\varphi-u) \cdot \nu|}\right) d H^{N-1} \\
= & \int_{\Omega} h(x, D u)+\int_{\partial \Omega}|\varphi-u| f^{0}(x, \nu(x)) d H^{N-1},
\end{aligned}
$$

(3.57) follows from (3.52) and (3.59).

By (3.49), (3.50) and (3.48), applying Lemma 3.6 (ii), we get

$$
|[z(x), \nu(x)]| \leq f^{0}(x, \nu(x)) \quad \text { a.e. in } \partial \Omega .
$$

Let $v_{j} \in C^{1}(\bar{\Omega})$ be a sequence such that $v_{j} \rightarrow u$ in $L^{2}(\Omega)$ and $\int_{\Omega}\left|\nabla v_{j}\right| \rightarrow$ $\|D u\|$. According to $\left(\mathrm{H}_{5}\right)$, we have

$$
\left|\mathbf{a}\left(x, \nabla u_{n}\right) \cdot \nabla v_{j}\right| \leq f^{0}\left(x, \nabla v_{j}\right) .
$$

Then, if $\psi, \phi \in C^{1}(\Omega)$, with $0 \leq \psi \leq \phi$, we have

$$
\left|\int_{\Omega} \mathbf{a}\left(x, \nabla u_{n}\right) \cdot \nabla v_{j} \psi d x\right| \leq \int_{\Omega} f^{0}\left(x, \nabla v_{j}\right) \psi d x,
$$

and, letting $n \rightarrow \infty$, we get

$$
\left|\int_{\Omega} z \cdot \nabla v_{j} \psi d x\right| \leq \int_{\Omega} f^{0}\left(x, \nabla v_{j}\right) \psi d x
$$

Now, since

$$
\left|\int_{\Omega} z \cdot \nabla v_{j} \psi d x\right|=\left|-\int_{\Omega} \operatorname{div}(z) v_{j} \psi d x-\int_{\Omega} v_{j} z \cdot \nabla \psi d x\right|,
$$


letting $j \rightarrow \infty$ we obtain that

$$
\begin{aligned}
|\langle(z, D u), \psi\rangle| & =\left|-\int_{\Omega} \operatorname{div}(z) u \psi d x-\int_{\Omega} u z \cdot \nabla \psi d x\right| \\
& \leq \int_{\Omega} \psi f^{0}(x, D u) \leq \int_{\Omega} \phi f^{0}(x, D u) .
\end{aligned}
$$

Hence

$$
\langle|(z, D u)|, \phi\rangle \leq \int_{\Omega} \phi f^{0}(x, D u) .
$$

Thus, we have

$$
|(z, D u)| \leq f^{0}(x, D u) \quad \text { as measures in } \Omega
$$

Then, the singular parts also satisfy a similar inequality,

$$
\left|z \cdot D^{s} u\right| \leq f^{0}\left(x, D^{s} u\right) \quad \text { as measures in } \Omega .
$$

Now, by (3.58), (3.51), (3.60) and (3.61), the assumptions of Lemma 3.5 are satisfied, and we have

$$
\begin{gathered}
\int_{\Omega} z \cdot \nabla u d x=\int_{\Omega} h(x, \nabla u) d x=\int_{\Omega} \mathbf{a}(x, \nabla u) \cdot \nabla u d x \\
z \cdot D^{s} u=f^{0}\left(x, D^{s} u\right), \\
{[z, \nu] \in \operatorname{sign}(\varphi-u) f^{0}(x, \nu(x)) \quad H^{N-1}-\text { a.e. }}
\end{gathered}
$$

Moreover, since the assumptions of Lemma 3.7 hold, we have that

$$
z(x)=\mathbf{a}(x, \nabla u(x)) \quad \text { a.e. } x \in \Omega .
$$

Observe that (3.39) follows from (3.47) and (3.65); (3.40) is a consequence of (3.62), (3.63) and (3.65); and (3.41) follows from (3.64) and (3.65). This concludes the proof in the case $\varphi \in C^{1}(\bar{\Omega})$.

Step 2. Suppose now we are in the general case, that is, $\varphi \in L^{1}(\partial \Omega)$. Take $\varphi_{j} \in C^{1}(\bar{\Omega})$ such that $\varphi_{j} \rightarrow \varphi$ in $L^{1}(\partial \Omega)$. Given $v \in L^{\infty}(\Omega)$, from the Step 1 , there exists $u_{j} \in D\left(\mathcal{B}_{\varphi_{j}}\right)$ such that $\left(u_{j}, v-u_{j}\right) \in \mathcal{B}_{\varphi_{j}}$. Hence, we have

$$
\begin{gathered}
-\operatorname{div}\left(\mathbf{a}\left(x, \nabla u_{j}\right)\right)=v-u_{j}, \quad \text { in } \quad \mathcal{D}^{\prime}(\Omega), \\
\mathbf{a}\left(x, \nabla u_{j}\right) \cdot D^{s} u_{j}=f^{0}\left(x, D^{s} u_{j}\right), \\
{\left[\mathbf{a}\left(x, \nabla u_{j}\right), \nu\right] \in \operatorname{sign}\left(\varphi_{j}-u_{j}\right) f^{0}(x, \nu(x)) \quad H^{N-1}-\text { a.e. }}
\end{gathered}
$$


By (3.66), (3.67) and (3.68), we get

$$
\begin{aligned}
& \int_{\Omega} \mathbf{a}\left(x, \nabla u_{j}\right) \cdot \nabla u_{j} d x+\int_{\Omega} f^{0}\left(x, D^{s} u_{j}\right) \\
& \quad+\int_{\partial \Omega}\left|\varphi_{j}-u_{j}\right| f^{0}(x, \nu(x)) d H^{N-1}+\int_{\Omega} u_{j}^{2} d x \\
& \quad=\int_{\Omega} u_{j} v d x+\int_{\partial \Omega}\left(\mathbf{a}\left(x, \nabla u_{j}\right) \cdot \nu\right) \varphi_{j} d H^{N-1} .
\end{aligned}
$$

From (3.69), using Young's inequality and (2.5), we obtain that

$$
C_{0}\left\|D u_{j}\right\|+C_{0} \int_{\partial \Omega}\left|\varphi_{j}-u_{j}\right| f^{0}(x, \nu(x)) d H^{N-1}+\frac{1}{2} \int_{\Omega} u_{j}^{2} d x \leq C \quad \forall j \in \mathbb{N},
$$

for some constant $C>0$. It follows that there exists $u \in B V(\Omega) \cap L^{2}(\Omega)$, such that

$$
\begin{array}{ll}
u_{j} \rightarrow u & \text { weakly in } L^{2}(\Omega), \\
u_{j} \rightarrow u & \text { in } \quad L^{q}(\Omega) \text { for all } 1 \leq q<\frac{N}{N-1}
\end{array}
$$

Hence,

$$
\int_{\Omega} u^{2} d x \leq \limsup _{j \rightarrow \infty} \int_{\Omega} u_{j}^{2} d x
$$

After passing to a subsequence, if necessary, we may assume that

$$
\mathbf{a}\left(x, \nabla u_{j}\right) \rightarrow z \quad \text { as } j \rightarrow \infty, \text { weakly* in } L^{\infty}\left(\Omega, \mathbb{R}^{N}\right)
$$

and

$$
-\operatorname{div}(z)=v-u \quad \text { in } \quad \mathcal{D}^{\prime}(\Omega) .
$$

According to [6], Fact 3.3, there exists a sequence $\left\{w_{k}\right\} \subset C^{1}(\Omega) \cap B V(\Omega)$ such that $w_{k \mid \partial \Omega}=\varphi$,

$$
w_{k} \rightarrow u \text { in } L^{2}(\Omega) \quad \text { and } \quad \Phi_{\varphi}\left(w_{k}\right) \rightarrow \Phi_{\varphi}(u) .
$$

Now, by the convexity of $f$ we have

$$
\begin{aligned}
\int_{\Omega} f\left(x, \nabla u_{j}\right) d x \leq & \int_{\Omega} \mathbf{a}\left(x, \nabla u_{j}\right) \cdot \nabla u_{j} d x-\int_{\Omega} \mathbf{a}\left(x, \nabla u_{j}\right) \cdot \nabla w_{k} d x \\
& +\int_{\Omega} f\left(x, \nabla w_{k}\right) d x
\end{aligned}
$$


Thus, having in mind (3.66), (3.67) and (3.68), we get

$$
\begin{aligned}
\Phi_{\varphi_{j}}\left(u_{j}\right) & =\int_{\Omega} f\left(x, \nabla u_{j}\right) d x+\int_{\Omega} f^{0}\left(x, D^{s} u_{j}\right)+\int_{\partial \Omega}\left|u_{j}-\varphi_{j}\right| f^{0}(x, \nu(x)) d H^{N-1} \\
\leq & \int_{\Omega} f\left(x, \nabla w_{k}\right) d x+\int_{\Omega} \mathbf{a}\left(x, \nabla u_{j}\right) \cdot \nabla u_{j} d x+\int_{\Omega} f^{0}\left(x, D^{s} u_{j}\right)+ \\
& +\int_{\partial \Omega}\left|u_{j}-\varphi_{j}\right| f^{0}(x, \nu(x)) d H^{N-1}-\int_{\Omega} \mathbf{a}\left(x, \nabla u_{j}\right) \cdot \nabla w_{k} d x \\
\leq & \int_{\Omega} f\left(x, \nabla w_{k}\right) d x+\int_{\Omega}\left(v-u_{j}\right) u_{j} d x \\
& +\int_{\partial \Omega}\left[\mathbf{a}\left(x, \nabla u_{j}\right), \nu\right] \varphi_{j} d H^{N-1}-\int_{\Omega} \mathbf{a}\left(x, \nabla u_{j}\right) \cdot \nabla w_{k} d x .
\end{aligned}
$$

Using (3.71) and (3.72), it follows that

$$
\begin{gathered}
\limsup _{j \rightarrow \infty} \Phi_{\varphi}\left(u_{j}\right)=\limsup _{j \rightarrow \infty} \Phi_{\varphi_{j}}\left(u_{j}\right) \leq \\
\leq \int_{\Omega} f\left(x, \nabla w_{k}\right) d x+\int_{\Omega} u v d x-\int_{\Omega} u^{2} d x+\int_{\partial \Omega}[z, \nu] \varphi d H^{N-1}-\int_{\Omega} z \cdot \nabla w_{k} d x \\
\leq \int_{\Omega} f\left(x, \nabla w_{k}\right) d x+\int_{\Omega}(v-u) u d x+\int_{\Omega} \operatorname{div}(z) w_{k} d x .
\end{gathered}
$$

Hence, by (3.73), letting $k \rightarrow \infty$, we arrive to

$$
\limsup _{j \rightarrow \infty} \Phi_{\varphi}\left(u_{j}\right) \leq \lim _{k \rightarrow \infty} \Phi_{\varphi}\left(w_{k}\right)=\Phi_{\varphi}(u) .
$$

Thus, by the lower-semicontinuity of $\Phi_{\varphi}$, we get

$$
\Phi_{\varphi}(u)=\lim _{j \rightarrow \infty} \Phi_{\varphi}\left(u_{j}\right) .
$$

Applying Theorem 3 of [18] as in the Step 1, it follows that

$$
\begin{aligned}
\lim _{j \rightarrow \infty} & \int_{\Omega} h\left(x, D u_{j}\right)+\int_{\partial \Omega}\left|u_{j}-\varphi_{j}\right| f^{0}(x, \nu(x)) d H^{N-1} \\
& =\int_{\Omega} h(x, D u)+\int_{\partial \Omega}|u-\varphi| f^{0}(x, \nu(x)) d H^{N-1} .
\end{aligned}
$$

On the other hand, by Green's formula, (3.66), (3.67) and (3.68), we have

$$
\begin{aligned}
& \int_{\Omega} h\left(x, D u_{j}\right)+\int_{\partial \Omega}\left|u_{j}-\varphi_{j}\right| f^{0}(x, \nu(x)) d H^{N-1} \\
& =\int_{\Omega}\left(\mathbf{a}\left(x, \nabla u_{j}\right), D u_{j}\right)+\int_{\partial \Omega}\left[\mathbf{a}\left(x, \nabla u_{j}\right), \nu\right]\left(\varphi_{j}-u_{j}\right) d H^{N-1} \\
& =\int_{\Omega} u_{j}\left(v-u_{j}\right) d x+\int_{\partial \Omega}\left[\mathbf{a}\left(x, \nabla u_{j}\right), \nu\right] \varphi_{j} d H^{N-1} .
\end{aligned}
$$


Since $\left[\mathbf{a}\left(x, \nabla u_{j}\right), \nu\right] \rightarrow[z, \nu]$ weakly* in $L^{\infty}(\partial \Omega)$, letting $j \rightarrow+\infty$, and using (3.76), it follows that

$$
\begin{aligned}
\int_{\Omega} h(x, D u)+ & \int_{\partial \Omega}|u-\varphi| f^{0}(x, \nu(x)) d H^{N-1} \\
& \leq \int_{\Omega} u(v-u) d x+\int_{\partial \Omega}[z, \nu] \varphi d H^{N-1} \\
& =\int_{\Omega}(z, D u)+\int_{\partial \Omega}[z, \nu](\varphi-u) d H^{N-1}
\end{aligned}
$$

Now, by Lemma 3.6 (i), we have

$$
|[z(x), \nu(x)]| \leq f^{0}(x, \nu(x)) \quad H^{N-1}-a . e \text { in } \partial \Omega .
$$

Moreover, as in the Step 1, we get

$$
\left|z \cdot D^{s} u\right| \leq f^{0}\left(x, D^{s} u\right) \quad \text { as measures in } \Omega
$$

With this and using Lemma 3.5, we obtain

$$
\begin{gathered}
\int_{\Omega} z \cdot \nabla u d x=\int_{\Omega} h(x, \nabla u) d x=\int_{\Omega} \mathbf{a}(x, \nabla u) \cdot \nabla u d x, \\
z \cdot D^{s} u=f^{0}\left(x, D^{s} u\right), \\
{[z, \nu] \in \operatorname{sign}(\varphi-u) f^{0}(x, \nu(x)) \quad H^{N-1}-\text { a.e. }}
\end{gathered}
$$

As in the Step 1 , to get that $(u, v-u) \in \mathcal{B}_{\varphi}$, we only need to prove that

$$
\operatorname{div} z=\operatorname{div} \mathbf{a}(x, \nabla u), \quad \text { in } \quad \mathcal{D}^{\prime}(\Omega),
$$

and

$$
[z, \nu]=[\mathbf{a}(x, \nabla u), \nu] \quad H^{N-1}-\text { a.e. on } \partial \Omega \text {. }
$$

Now, by (3.66), (3.70) and using Fatou's Lemma, we are able to adapt the proof of Lemma 3.7 obtaining that $z(x)=\mathbf{a}(x, \nabla u(x))$ a.e. in $\Omega$ and this implies both (3.80) and (3.81).

Step 3. To prove the density of $D\left(\mathcal{B}_{\varphi}\right)$ in $L^{2}(\Omega)$, we prove that $C_{0}^{\infty}(\Omega) \subseteq$ ${\overline{D\left(\mathcal{B}_{\varphi}\right)}}^{L^{2}(\Omega)}$. Let $v \in C_{0}^{\infty}(\Omega)$. By the above, $v \in R\left(I+\frac{1}{n} \mathcal{B}_{\varphi}\right)$ for all $n \in \mathbb{N}$. Thus, for each $n \in \mathbb{N}$, there exists $u_{n} \in D\left(\mathcal{B}_{\varphi}\right)$ such that $\left(u_{n}, n\left(v-u_{n}\right)\right) \in \mathcal{B}_{\varphi}$. Consequently, we have $\mathbf{a}\left(x, \nabla u_{n}\right) \in X(\Omega), n\left(v-u_{n}\right)=-\operatorname{div}\left(\mathbf{a}\left(x, \nabla u_{n}\right)\right)$ in 
$\mathcal{D}^{\prime}(\Omega)$ and

$$
\begin{aligned}
& \int_{\Omega}\left(w-u_{n}\right) n\left(v-u_{n}\right) d x= \\
& =\int_{\Omega}\left(\mathbf{a}\left(x, \nabla u_{n}\right), D w\right)-\int_{\partial \Omega}\left[\mathbf{a}\left(x, \nabla u_{n}\right), \nu\right](w-\varphi) d H^{N-1} \\
& \quad-\int_{\Omega} h\left(x, D u_{n}\right)-\int_{\partial \Omega}\left|\varphi-u_{n}\right| f^{0}(x, \nu(x)) d H^{N-1} .
\end{aligned}
$$

for every $w \in B V(\Omega) \cap L^{2}(\Omega)$. Taking $w=v$, we get

$$
\begin{aligned}
\int_{\Omega}\left(v-u_{n}\right)^{2} d x & =\frac{1}{n}\left(\int_{\Omega} \mathbf{a}\left(x, \nabla u_{n}\right) \cdot \nabla v d x-\int_{\partial \Omega}\left[\mathbf{a}\left(x, \nabla u_{n}\right), \nu\right](v-\varphi) d H^{N-1}\right. \\
& \left.-\int_{\Omega} h\left(x, D u_{n}\right)-\int_{\partial \Omega}\left|\varphi-u_{n}\right| f^{0}(x, \nu(x)) d H^{N-1}\right) \\
\leq & \frac{1}{n}\left(\int_{\Omega} \mathbf{a}\left(x, \nabla u_{n}\right) \cdot \nabla v d x-\int_{\partial \Omega}\left[\mathbf{a}\left(x, \nabla u_{n}\right), \nu\right](v-\varphi) d H^{N-1}\right) \\
\leq & \frac{M}{n}\left(\int_{\Omega}|\nabla v| d x+\int_{\partial \Omega}|v-\varphi| d H^{N-1}\right) .
\end{aligned}
$$

Letting $n \rightarrow \infty$, it follows that $u_{n} \rightarrow v$ in $L^{2}(\Omega)$. Therefore $v \in \overline{D\left(\mathcal{B}_{\varphi}\right)} L^{2}(\Omega)$ and the proof is complete.

Proof of Theorem 3.1 First, we prove that $\mathcal{B}_{\varphi} \subset \partial \Phi_{\varphi}$. Let $(u, v) \in \mathcal{B}_{\varphi}$ and $w \in W_{\varphi}^{1,2}(\Omega)$. Then, by (2.2), and applying Green's formula we get

$$
\begin{aligned}
& \int_{\Omega}(w-u) v d x=-\int_{\Omega}(w-u) d i v \mathbf{a}(x, \nabla u) d x \\
& =\int_{\Omega}(\mathbf{a}(x, \nabla u), D w-D u)-\int_{\partial \Omega}[\mathbf{a}(x, \nabla u), \nu](\varphi-u) d H^{N-1} \\
& =\int_{\Omega} \mathbf{a}(x, \nabla u) \cdot \nabla w d x-\int_{\Omega} \mathbf{a}(x, \nabla u) \cdot \nabla u d x-\int_{\Omega} \mathbf{a}(x, \nabla u) \cdot D^{s} u \\
& \quad-\int_{\partial \Omega}[\mathbf{a}(x, \nabla u), \nu](\varphi-u) d H^{N-1} \\
& \leq \int_{\Omega} f(x, \nabla w) d x-\int_{\Omega} f(x, D u) d x-\int_{\partial \Omega}|\varphi-u| f^{0}(x, \nu(x)) d H^{N-1} \\
& =\Phi_{\varphi}(w)-\Phi_{\varphi}(u) .
\end{aligned}
$$

Suppose that $w \in B V(\Omega) \cap L^{2}(\Omega)$. According to [6], Fact 3.3, there exists a sequence $w_{n} \in W_{\varphi}^{1,2}(\Omega)$, with $w_{n} \rightarrow w$ in $L^{2}(\Omega)$, and $\Phi_{\varphi}\left(w_{n}\right) \rightarrow \Phi_{\varphi}(w)$. 
Then, by the above inequality, we have

$$
\int_{\Omega}\left(w_{n}-u\right) v d x \leq \Phi_{\varphi}\left(w_{n}\right)-\Phi_{\varphi}(u) .
$$

Now, letting $n \rightarrow \infty$, we get

$$
\int_{\Omega}(w-u) v d x \leq \Phi_{\varphi}(w)-\Phi_{\varphi}(u)
$$

and therefore, $(u, v) \in \partial \Phi_{\varphi}$.

Since $\mathcal{B}_{\varphi} \subset \partial \Phi_{\varphi}$, and, by Proposition $3.2, L^{\infty}(\Omega) \subset R\left(I+\mathcal{B}_{\varphi}\right)$, we have $\partial \Phi_{\varphi}=\overline{\mathcal{B}}_{\varphi}{ }^{2}(\Omega)$. To finish the proof we only need to prove that the operator $\mathcal{B}_{\varphi}$ is closed. Let $\left(u_{n}, v_{n}\right) \in \mathcal{B}_{\varphi}$, and assume that $\left(u_{n}, v_{n}\right) \rightarrow(u, v)$ in $L^{2}(\Omega) \times L^{2}(\Omega)$. Let us prove that $(u, v) \in \mathcal{B}_{\varphi}$. Since $\left(u_{n}, v_{n}\right) \in \mathcal{B}_{\varphi}$, we know that $\mathbf{a}\left(x, \nabla u_{n}\right) \in X(\Omega)$ is such that

$$
\begin{gathered}
-v_{n}=\operatorname{div} \mathbf{a}\left(x, \nabla u_{n}\right), \quad \text { in } \mathcal{D}^{\prime}(\Omega), \\
\mathbf{a}\left(x, \nabla u_{n}\right) \cdot D^{s} u_{n}=f^{0}\left(x, D^{s} u_{n}\right), \\
{\left[\mathbf{a}\left(x, \nabla u_{n}\right), \nu\right] \in \operatorname{sign}\left(\varphi-u_{n}\right) f^{0}(x, \nu(x)) \quad H^{N-1}-\text { a.e. }}
\end{gathered}
$$

Multiplying (3.82) by $u_{n}$ and applying Green's formula we obtain

$$
\begin{aligned}
-\int_{\Omega} u_{n} v_{n} d x= & \int_{\partial \Omega}\left[\mathbf{a}\left(x, \nabla u_{n}\right), \nu\right] \varphi d H^{N-1}-\int_{\Omega} h\left(x, D u_{n}\right) \\
& -\int_{\partial \Omega}\left|\varphi-u_{n}\right| f^{0}(x, \nu(x)) d H^{N-1}
\end{aligned}
$$

Hence,

$$
\int_{\Omega} h\left(x, D u_{n}\right) \leq \int_{\Omega} u_{n} v_{n} d x+\int_{\partial \Omega}\left[\mathbf{a}\left(x, \nabla u_{n}\right), \nu\right] \varphi d H^{N-1} .
$$

From (2.5) and (3.85), we have

$$
\begin{aligned}
C_{0} \int_{\Omega}\left|D u_{n}\right| d x-D_{1} \lambda_{N}(\Omega) & \leq \int_{\Omega} h\left(x, D u_{n}\right) d x \\
& \leq \int_{\Omega} u_{n} v_{n} d x+\int_{\partial \Omega}\left[\mathbf{a}\left(x, \nabla u_{n}\right), \nu\right] \varphi d H^{N-1}
\end{aligned}
$$

Hence,

$$
\int_{\Omega}\left|D u_{n}\right| d x \leq M_{1} \quad \forall n \in \mathbb{N}
$$


Therefore, $u \in B V(\Omega) \cap L^{2}(\Omega)$. On the other hand, since $\left\|\mathbf{a}\left(x, \nabla u_{n}\right)\right\|_{\infty} \leq$ $M$, we may assume that

$$
\mathbf{a}\left(x, \nabla u_{n}\right) \rightarrow z \quad \text { in the weak }{ }^{*} \text { topology of } L^{\infty}\left(\Omega, \mathbb{R}^{N}\right),
$$

with $\|z\|_{\infty} \leq M$. Moreover, since $v_{n} \rightarrow v$ in $L^{2}(\Omega)$, we have that $v=$ $-\operatorname{div}(z)$ in $\mathcal{D}^{\prime}(\Omega)$. By the definition of the weak trace on $\partial \Omega$ of the normal component of $z$, it is easy to see that

$$
\left[\mathbf{a}\left(x, \nabla u_{n}\right), \nu\right] \rightarrow[z, \nu] \quad \text { weakly }^{*} \text { in } L^{\infty}(\partial \Omega) .
$$

Now, we prove the convergence of the energies. According to [6], Fact 3.3 , there exists a sequence $w_{j} \in C^{1}(\Omega) \cap B V(\Omega)$, with $w_{j \mid \partial \Omega}=\varphi, w_{j} \rightarrow u$ in $L^{1}(\Omega)$, and $\Phi_{\varphi}\left(w_{j}\right) \rightarrow \Phi_{\varphi}(u)$. Moreover, looking at the proof of Fact 3.3 in [6], we have that, $w_{j}=w_{j}^{1}+w_{j}^{2}$ with $w_{j \mid \partial \Omega}^{1}=u_{\mid \partial \Omega}$ and $w_{j}^{1} \rightarrow u$ in $L^{1}(\Omega)$, $w_{j \mid \partial \Omega}^{2}=\varphi-u_{\mid \partial \Omega}, w_{j}^{2} \rightarrow 0$ in $L^{1}(\Omega)$, and, using [5], Lemma 1.8, we have that

$$
\int_{\Omega}\left(z, D w_{j}^{1}\right) \rightarrow \int_{\Omega}(z, D u)
$$

By the convexity of $f$ and taking (3.83) and (3.84) into account we have

$$
\begin{aligned}
& \Phi_{\varphi}\left(u_{n}\right)=\int_{\Omega} f\left(x, \nabla u_{n}\right) d x+\int_{\Omega} f^{0}\left(x, D^{s} u_{n}\right)+\int_{\partial \Omega}\left|u_{n}-\varphi\right| f^{0}(x, \nu(x)) d H^{N-1} \\
\leq & \int_{\Omega} \mathbf{a}\left(x, \nabla u_{n}\right) \cdot \nabla u_{n} d x-\int_{\Omega} \mathbf{a}\left(x, \nabla u_{n}\right) \cdot \nabla w_{j} d x+\int_{\Omega} f\left(x, \nabla w_{j}\right) d x \\
& +\int_{\Omega} \mathbf{a}\left(x, \nabla u_{n}\right) \cdot D^{s} u_{n}+\int_{\partial \Omega}\left[\mathbf{a}\left(x, \nabla u_{n}\right), \nu\right]\left(\varphi-u_{n}\right) d H^{N-1} \\
= & \int_{\Omega}\left(\mathbf{a}\left(x, \nabla u_{n}\right), D u_{n}\right)-\int_{\Omega} \mathbf{a}\left(x, \nabla u_{n}\right) \cdot \nabla w_{j} d x+\Phi_{\varphi}\left(w_{j}\right) \\
& +\int_{\partial \Omega}\left[\mathbf{a}\left(x, \nabla u_{n}\right), \nu\right]\left(\varphi-u_{n}\right) d H^{N-1}=\Phi_{\varphi}\left(w_{j}\right)-\int_{\Omega} \mathbf{a}\left(x, \nabla u_{n}\right) \cdot \nabla w_{j} d x \\
& -\int_{\Omega} d i v\left(\mathbf{a}\left(x, \nabla u_{n}\right)\right) u_{n} d x+\int_{\partial \Omega}\left[\mathbf{a}\left(x, \nabla u_{n}\right), \nu\right] \varphi d H^{N-1} \\
= & \Phi_{\varphi}\left(w_{j}\right)-\int_{\Omega} \mathbf{a}\left(x, \nabla u_{n}\right) \cdot \nabla w_{j} d x+\int_{\Omega} v_{n} u_{n} d x+\int_{\partial \Omega}\left[\mathbf{a}\left(x, \nabla u_{n}\right), \nu\right] \varphi d H^{N-1} .
\end{aligned}
$$

Hence, by (3.87) and (3.88), it follows that

$$
\begin{aligned}
& \limsup _{n \rightarrow \infty} \Phi_{\varphi}\left(u_{n}\right) \leq \Phi_{\varphi}\left(w_{j}\right)-\int_{\Omega}\left(z, D w_{j}\right)+\int_{\Omega} u v d x+\int_{\partial \Omega}[z, \nu] \varphi d H^{N-1} \\
= & \Phi_{\varphi}\left(w_{j}\right)-\int_{\Omega}\left(z, D w_{j}^{1}\right)-\int_{\Omega}\left(z, D w_{j}^{2}\right)-\int_{\Omega} \operatorname{div}(z) u d x+\int_{\partial \Omega}[z, \nu] \varphi d H^{N-1}
\end{aligned}
$$


$=\Phi_{\varphi}\left(w_{j}\right)-\int_{\Omega}\left(z, D w_{j}^{1}\right)+\int_{\Omega} \operatorname{div}(z) w_{j}^{2} d x+\int_{\partial \Omega}[z, \nu] u d H^{N-1}-\int_{\Omega} \operatorname{div}(z) u d x$.

Letting $j \rightarrow \infty$, we have that

$$
\begin{aligned}
\limsup _{n \rightarrow \infty} \Phi_{\varphi}\left(u_{n}\right) & \leq \Phi_{\varphi}(u)-\int_{\Omega}(z, D u)+\int_{\partial \Omega}[z, \nu] u d H^{N-1}-\int_{\Omega} \operatorname{div}(z) u d x \\
& =\Phi_{\varphi}(u) .
\end{aligned}
$$

Finally, by the lower-semicontinuity of $\Phi_{\varphi}$, we obtain

$$
\Phi_{\varphi}(u)=\lim _{n \rightarrow \infty} \Phi_{\varphi}\left(u_{n}\right)
$$

If we consider the $\mathbb{R}^{N}$-valued measures $\mu_{n}, \mu$ on $\bar{\Omega}$ which are defined as

$$
\begin{aligned}
\mu_{n}(B) & :=\int_{B \cap \Omega} D u_{n}+\int_{B \cap \partial \Omega}\left(\varphi-u_{n}\right) \nu d H^{N-1}, \\
\mu(B) & :=\int_{B \cap \Omega} D u+\int_{B \cap \partial \Omega}(\varphi-u) \nu d H^{N-1}
\end{aligned}
$$

for all Borel sets $B \subset \bar{\Omega}$, we have

$$
\mu_{j} \rightarrow \mu \quad \text { weakly as measures in } \bar{\Omega} \text {. }
$$

Moreover,

$$
\Phi_{\varphi}(u)=\int_{\bar{\Omega}} \tilde{f}(x, \tilde{\mu}) \quad \text { and } \quad \Phi_{\varphi}\left(u_{n}\right)=\int_{\bar{\Omega}} \tilde{f}\left(x, \tilde{\mu}_{n}\right)
$$

Hence, (3.89) yields

$$
\lim _{n \rightarrow \infty} \int_{\bar{\Omega}} \tilde{f}\left(x, \tilde{\mu}_{n}\right)=\int_{\bar{\Omega}} \tilde{f}(x, \tilde{\mu}) .
$$

Then, applying [18], Theorem 3, it follows that

$$
\begin{aligned}
\int_{\bar{\Omega}} \tilde{h}(x, \tilde{\mu}) & =\lim _{n \rightarrow \infty} \int_{\bar{\Omega}} \tilde{h}\left(x, \tilde{\mu}_{n}\right) \\
& =\lim _{n \rightarrow \infty} \int_{\Omega} h\left(x, D u_{n}\right)+\int_{\partial \Omega}\left|u_{n}-\varphi\right| f^{0}(x, \nu(x)) d H^{N-1} .
\end{aligned}
$$

Since

$$
\int_{\bar{\Omega}} \tilde{h}(x, \tilde{\mu})=\int_{\Omega} h(x, D u)+\int_{\partial \Omega}|\varphi-u| f^{0}(x, \nu(x)) d H^{N-1}
$$


we have

$$
\begin{aligned}
& \int_{\Omega} h(x, D u)+\int_{\partial \Omega}|\varphi-u| f^{0}(x, \nu(x)) d H^{N-1}= \\
& =\lim _{n \rightarrow \infty} \int_{\Omega} h\left(x, D u_{n}\right)+\int_{\partial \Omega}\left|u_{n}-\varphi\right| f^{0}(x, \nu(x)) d H^{N-1} .
\end{aligned}
$$

Now, since

$$
\begin{aligned}
& \lim _{n \rightarrow \infty} \int_{\Omega} h\left(x, D u_{n}\right)+\int_{\partial \Omega}\left|u_{n}-\varphi\right| f^{0}(x, \nu(x)) d H^{N-1} \\
= & \lim _{n \rightarrow \infty} \int_{\Omega} \mathbf{a}\left(x, \nabla u_{n}\right) \cdot \nabla u_{n} d x+\int_{\Omega} f^{0}\left(x, D^{s} u_{n}\right) \\
& +\int_{\partial \Omega}\left[\mathbf{a}\left(x, \nabla u_{n}\right), \nu\right]\left(\varphi-u_{n}\right) d H^{N-1} \\
= & \lim _{n \rightarrow \infty} \int_{\Omega}\left(\mathbf{a}\left(x, \nabla u_{n}\right), D u_{n}\right)+\int_{\partial \Omega}\left[\mathbf{a}\left(x, \nabla u_{n}\right), \nu\right]\left(\varphi-u_{n}\right) d H^{N-1} \\
= & \lim _{n \rightarrow \infty} \int_{\partial \Omega}\left[\mathbf{a}\left(x, \nabla u_{n}\right), \nu\right] \varphi d H^{N-1}-\int_{\Omega} \operatorname{div}\left(\mathbf{a}\left(x, \nabla u_{n}\right)\right) u_{n} d x \\
= & \int_{\partial \Omega}[z, \nu] \varphi d H^{N-1}-\int_{\Omega} d i v(z) u d x \\
= & \int_{\Omega}(z, D u)+\int_{\partial \Omega}[z, \nu](\varphi-u) d H^{N-1},
\end{aligned}
$$

we finally obtain

$$
\begin{aligned}
\int_{\Omega} h(x, D u) & +\int_{\partial \Omega}|\varphi-u| f^{0}(x, \nu(x)) d H^{N-1} \\
& =\int_{\Omega}(z, D u)+\int_{\partial \Omega}[z, \nu](\varphi-u) d H^{N-1} .
\end{aligned}
$$

Again, by (3.87) and (3.88) we can apply Lemma 3.6 obtaining that

$$
|[z(x), \nu(x)]| \leq f^{0}(x, \nu(x)) \quad \text { a.e. in } \partial \Omega .
$$

Moreover, acting as in the proof of Proposition 3.2, we get that

$$
\left|z \cdot D^{s} u\right| \leq f^{0}\left(x, D^{s} u\right) \quad \text { as measures in } \Omega \text {. }
$$

Hence by (3.89), (3.90), (3.91), (3.87) (3.93) and (3.92), we can apply Lemma 3.5, to obtain

$$
\begin{gathered}
\int_{\Omega} z \cdot \nabla u d x=\int_{\Omega} h(x, \nabla u) d x=\int_{\Omega} \mathbf{a}(x, \nabla u) \cdot \nabla u d x \\
z \cdot D^{s} u=f^{0}\left(x, D^{s} u\right), \\
{[z, \nu] \in \operatorname{sign}(\varphi-u) f^{0}(x, \nu(x)) \quad H^{N-1}-\text { a.e. }}
\end{gathered}
$$


Now, using Lemma 3.7, we have

$$
\operatorname{div} z=\operatorname{div} \mathbf{a}(x, \nabla u), \quad \text { in } \mathcal{D}^{\prime}(\Omega),
$$

and

$$
[z, \nu]=[\mathbf{a}(x, \nabla u), \nu] \quad H^{N-1}-\text { a.e. on } \partial \Omega .
$$

Since $v=-\operatorname{div}(z)$ in $\mathcal{D}^{\prime}(\Omega)$, taking (3.97) into account, we get

$$
v=-\operatorname{div}(\mathbf{a}(x, \nabla u)), \quad \text { in } \mathcal{D}^{\prime}(\Omega),
$$

and, using (3.94), (3.95) and (3.97), we get

$$
\mathbf{a}(x, \nabla u) \cdot D^{s} u=f^{0}\left(x, D^{s} u\right) .
$$

Finally, by (3.96) and (3.98) we get

$$
[\mathbf{a}(x, \nabla u), \nu] \in \operatorname{sign}(\varphi-u) f^{0}(x, \nu(x)) \quad H^{N-1}-\text { a.e. }
$$

Therefore, $(u, v) \in \mathcal{B}_{\varphi}$.

Proof of Theorem 2.2 Let $(S(t))_{t \geq 0}$ be the semigroup in $L^{2}(\Omega)$ generated by the subdifferential of $\Phi_{\varphi}$. Then by the nonlinear semigroup theory ([9]), given $u_{0} \in L^{2}(\Omega)=\overline{D\left(\partial \Phi_{\varphi}\right)}, u(t)=S(t) u_{0}$ is the only strong solution of problem (3.1). Thus, by Theorem 3.1, we have that for almost all $t \in$ $\left[0,+\infty\left[, u(t) \in D\left(\mathcal{B}_{\varphi}\right)\right.\right.$ and $-u^{\prime}(t) \in \mathcal{B}_{\varphi}(u(t))$. This concludes the proof.

\section{Behaviour of the solution}

We have the following weak form of the maximum principle.

Theorem 4.1 Suppose $u_{1}$ and $u_{2}$ are two solutions of (1.1) corresponding to initial data $u_{1,0}$ and $u_{2,0}$ in $L^{2}(\Omega)$ and boundary data $\varphi_{1}$ and $\varphi_{2}$ in $L^{1}(\partial \Omega)$, respectively. If

$$
u_{1,0} \geq u_{2,0} \quad \text { and } \quad \varphi_{1} \geq \varphi_{2}
$$

then $u_{1} \geq u_{2}$.

Proof. For almost all $t \in\left[0,+\infty\left[\right.\right.$, we have $u_{i}^{\prime}(t) \in L^{2}(\Omega), u_{i}(t) \in B V(\Omega) \cap$ $L^{2}(\Omega), \mathbf{a}\left(x, \nabla u_{i}(t)\right) \in X(\Omega)$, and

$$
\begin{gathered}
u_{2}^{\prime}(t)-u_{1}^{\prime}(t)=\operatorname{div}\left[\mathbf{a}\left(x, \nabla u_{2}(t)\right)-\mathbf{a}\left(x, \nabla u_{1}(t)\right)\right], \quad \text { in } \mathcal{D}^{\prime}(\Omega), \\
\mathbf{a}\left(x, \nabla u_{i}(t)\right) \cdot D^{s} u_{i}(t)=f^{0}\left(x, D^{s} u_{i}(t)\right), \\
{\left[\mathbf{a}\left(x, \nabla u_{i}(t)\right), \nu\right] \in \operatorname{sign}\left(\varphi_{i}-u_{i}(t)\right) f^{0}(x, \nu(x)) \quad H^{N-1}-\text { a.e. on } \partial \Omega .}
\end{gathered}
$$


Multiplying in $(4.1)$ by $\left(u_{2}(t)-u_{1}(t)\right)^{+}$, integrating in $\Omega$, and using Green's formula, we get

$$
\begin{aligned}
& \frac{1}{2} \int_{\Omega} \frac{d}{d t}\left[\left(u_{2}(t)-u_{1}(t)\right)^{+}\right]^{2} d x= \\
& =\int_{\Omega} \operatorname{div}\left[\mathbf{a}\left(x, \nabla u_{2}(t)\right)-\mathbf{a}\left(x, \nabla u_{1}(t)\right)\right]\left(u_{2}(t)-u_{1}(t)\right)^{+} d x \\
& =-\int_{\Omega}\left(\mathbf{a}\left(x, \nabla u_{2}(t)\right)-\mathbf{a}\left(x, \nabla u_{1}(t)\right), D\left(\left(u_{2}(t)-u_{1}(t)\right)^{+}\right)\right)+ \\
& \quad+\int_{\partial \Omega}\left[\mathbf{a}\left(x, \nabla u_{2}(t)\right)-\mathbf{a}\left(x, \nabla u_{1}(t)\right), \nu\right]\left(u_{2}(t)-u_{1}(t)\right)^{+} d H^{N-1} .
\end{aligned}
$$

Now, by the chain rule for BV-functions ([2], [14], Lemma 1.2), there exists a scalar function $\eta(t)$, with $0 \leq \eta(t) \leq 1$, such that

$$
\begin{aligned}
& \int_{\Omega}\left(\mathbf{a}\left(x, \nabla u_{2}(t)\right)-\mathbf{a}\left(x, \nabla u_{1}(t)\right), D\left(\left(u_{2}(t)-u_{1}(t)\right)^{+}\right)\right)= \\
& =\int_{\left\{u_{2} \geq u_{1}\right\}}\left(\mathbf{a}\left(x, \nabla u_{2}(t)\right)-\mathbf{a}\left(x, \nabla u_{1}(t)\right) \cdot\left(\nabla u_{2}(t)-\nabla u_{1}(t)\right) d x+\right. \\
& \quad+\int_{\Omega} \eta(t)\left(\mathbf{a}\left(x, \nabla u_{2}(t)\right)-\mathbf{a}\left(x, \nabla u_{1}(t)\right) \cdot D^{s}\left(u_{2}(t)-u_{1}(t)\right) .\right.
\end{aligned}
$$

Observe that, by the monotonicity of $\mathbf{a},\left(\mathrm{H}_{5}\right)$ and (4.2), we have that

$$
\int_{\Omega}\left(\mathbf{a}\left(x, \nabla u_{2}(t)\right)-\mathbf{a}\left(x, \nabla u_{1}(t)\right), D\left(\left(u_{2}(t)-u_{1}(t)\right)^{+}\right)\right) \geq 0 .
$$

On the other hand, since $\varphi_{1} \geq \varphi_{2}$, from (4.3), it is easy to see that

$$
\int_{\partial \Omega}\left[\mathbf{a}\left(x, \nabla u_{2}(t)\right)-\mathbf{a}\left(x, \nabla u_{1}(t)\right), \nu\right]\left(u_{2}(t)-u_{1}(t)\right)^{+} d H^{N-1} \leq 0 .
$$

From (4.4), (4.5) and (4.6), it follows that

$$
\frac{1}{2} \int_{\Omega} \frac{d}{d t}\left[\left(u_{2}(t)-u_{1}(t)\right)^{+}\right]^{2} d x \leq 0 .
$$

Since $u_{1,0} \geq u_{2,0}$, we have $u_{1} \geq u_{2}$, and the proof is concluded.

We shall now prove that the solution $u(t)$ stabilizes as $t \rightarrow+\infty$ by converging to a solution of the steady-state problem. To do that, we follow the proof of Theorem 4.2 in [17]. 
Theorem 4.2 Suppose $u_{0} \in L^{2}(\Omega) \cap B V(\Omega)$ and $\varphi \in L^{\infty}(\partial \Omega)$. Then the solution $u(t)$ of (1.1) converges as $t \rightarrow+\infty$ to some limit $w \in \mathcal{B}_{\varphi}^{-1}(0)$ in the following sense:

$$
u(t) \rightarrow w \text { strongly in } L^{1}(\Omega) \text { and weakly in } L^{2}(\Omega) .
$$

Proof. Since $\mathcal{B}_{\varphi}$ is the subdifferential of $\Phi_{\varphi}$, by a classical result of Bruck ([10], Theorem 4), to prove the weak convergence in $L^{2}(\Omega)$, it is sufficient to prove that $\Phi_{\varphi}$ attains its minimum in $L^{2}(\Omega)$. In fact, let $\left\{u_{n}\right\}$ be a minimizing sequence for $\Phi_{\varphi}$. Without loss of generality, we may assume that $u_{n} \in B V(\Omega) \cap L^{2}(\Omega)$. Now, by approximation we may assume that $u_{n} \in W^{1,1}(\Omega) \cap L^{2}(\Omega)$. Denote by $J: \mathbb{R} \rightarrow \mathbb{R}$ the truncature function

$$
J(r):=\left\{\begin{array}{ccc}
-\|\varphi\|_{\infty} & \text { if } & r<-\|\varphi\|_{\infty} \\
r & \text { if } & |r| \leq\|\varphi\|_{\infty} \\
\|\varphi\|_{\infty} & \text { if } & r>\|\varphi\|_{\infty} .
\end{array}\right.
$$

If we take $w_{n}:=J \circ u_{n}, w_{n} \in W^{1,1}(\Omega) \cap L^{\infty}(\Omega)$, and using that $\left|J^{\prime}\right| \leq 1$, we have

$$
\begin{gathered}
\Phi_{\varphi}\left(w_{n}\right)=\int_{\Omega} f\left(x, \nabla w_{n}\right) d x+\int_{\partial \Omega}\left|w_{n}-\varphi\right| f^{0}(x, \nu(x)) d H^{N-1}= \\
=\int_{\left\{\left|u_{n}\right| \leq\|\varphi\|_{\infty}\right\}} f\left(x, \nabla u_{n}\right) d x+\int_{\partial \Omega}\left|J \circ u_{n}-J \circ \varphi\right| f^{0}(x, \nu(x)) d H^{N-1} \leq \\
\leq \int_{\Omega} f\left(x, \nabla u_{n}\right) d x+\int_{\partial \Omega}\left|u_{n}-\varphi\right| f^{0}(x, \nu(x)) d H^{N-1} .
\end{gathered}
$$

Thus, $\left\{w_{n}\right\}$ is still a minimizing sequence for $\Phi_{\varphi}$. Moreover, this sequence is bounded in $W^{1,1}(\Omega) \cap L^{\infty}(\Omega)$, hence, relatively compact in $L^{1}(\Omega)$. We may extract a subsequence converging in $L^{1}(\Omega)$ to some $\bar{u} \in L^{1}(\Omega) \cap B V(\Omega)$. Therefore,

$$
\Phi_{\varphi}(\bar{u})=\inf _{u \in L^{2}(\Omega)} \Phi_{\varphi}(u) .
$$

Then, by Bruck's result ([10], Theorem 4), there exists $w \in \mathcal{B}_{\varphi}^{-1}(0)$, such that $u(t) \rightarrow w$ weakly in $L^{2}(\Omega)$. Finally, we prove the strong convergence in $L^{1}(\Omega)$. Since $\left(u(t),-u^{\prime}(t)\right) \in \partial \Phi_{\varphi}$, using [9], Lemma 3.3, we have

$$
\frac{d}{d s} \Phi_{\varphi}(u(s))=-\int_{\Omega} u^{\prime}(s)^{2} d x \leq 0
$$

hence,

$$
\Phi_{\varphi}(u(t)) \leq \Phi_{\varphi}\left(u_{0}\right) \quad \forall t>0 .
$$

Thus, $\{u(t): t \geq 0\}$ is bounded in $B V(\Omega)$, and therefore relatively compact in $L^{1}(\Omega)$. The result follows. 


\section{Appendix}

In this appendix we prove the approximation Lemma. Before giving the proof, let us construct a substitute for the distance function to the boundary $d(., \partial \Omega)$. That construction would be unnecessary if $\partial \Omega$ would be of class $W^{2, \infty}([7])$. We follow the proof of Lemma 5.1 in [7] for $C^{2}$ domains.

If $\partial \Omega$ is a manifold of class $C^{1}$, then there is some $\varepsilon>0$ such that for all points $y \in \Omega$ such that $d(y, \Omega)<\varepsilon$ there is $z \in \partial \Omega$ and $t \in(0, \varepsilon)$ such that $y=z-t \nu(z), \nu(z)$ being the outer unit normal to $\partial \Omega$ at $z([11])$. In other words, $\Omega^{\varepsilon}:=\{x \in \Omega: x=y-t \nu(y), y \in \partial \Omega, t \in(0, \varepsilon)\}$ is open. Then there is a function $D \in C^{1}(\bar{\Omega})$ such that $D=0$ on $\partial \Omega, D>0$ on $\Omega$ and $\nabla D(x)=-\nu(x)$ for all $x \in \partial \Omega$. This is a consequence of Withney's extension Theorem ([15], p.48, [13], p.245). Indeed, since

$$
\nu(y) \frac{x-y}{|x-y|} \rightarrow 0 \text { as } x, y \rightarrow p, x \neq y, x, y \in \partial \Omega,
$$

by Withney's Theorem, we know that there exists a function $\tilde{D} \in C^{1}(\bar{\Omega})$ such that $\tilde{D}=0$ on $\partial \Omega$ and $\nabla \tilde{D}(x)=-\nu(x)$ for all $x \in \partial \Omega$. Now, let $y \in \partial \Omega$ and $t \in(0, \varepsilon)$. Using the mean value theorem, we know that

$$
\tilde{D}(y-t \nu(y))=\tilde{D}(y)-t \nabla \tilde{D}(y-s \nu(y)) \cdot \nu(y)=-t \nabla \tilde{D}(y-s \nu(y)) \cdot \nu(y) .
$$

Since $\tilde{D} \in C^{1}(\bar{\Omega})$, we have

$$
\tilde{D}(y-t \nu(y))=t(1+\omega(t))
$$

where $\omega(t)=o(1)$ as $t \rightarrow 0+$ and is the modulus of continuity of $\nabla \tilde{D}$. Without loss of generality we may assume that $\varepsilon>0$ is such that $\omega(t)<\frac{1}{2}$ for all $t \in(0, \varepsilon)$. In particular, we have that

$$
\tilde{D}(x)>0 \quad \text { for all } x \in \Omega^{\varepsilon} \text {. }
$$

We shall modify $\tilde{D}$ so that the modified function is $>0$ in $\Omega$. Let $\eta \in$ $C([0, \infty)), \eta(t)>0$, for all $t \in(0, \infty), \eta(t)=o(t)$ as $t \rightarrow 0+$. Let $\Omega_{1}$ be an open set, $\overline{\Omega_{1}} \subset \Omega$, with smooth boundary $\partial \Omega_{1} \subset \Omega^{\varepsilon}$ such that $0<\delta-\eta(\delta)<$ $\tilde{D}(x)<\delta+\eta(\delta)$ for all $x \in \partial \Omega_{1}$ for some $\delta>0$. Let $\Omega_{2}^{\prime}$ be an open set with smooth boundary such that $\overline{\Omega_{2}^{\prime}} \subset \Omega_{1}$ and $\eta(\delta)<d\left(\partial \Omega_{1}, \partial \Omega_{2}^{\prime}\right)<2 \eta(\delta)$, where $d\left(\partial \Omega_{1}, \partial \Omega_{2}^{\prime}\right)=\inf \left\{|x-y|: x \in \partial \Omega_{1}, y \in \partial \Omega_{2}^{\prime}\right\}$. Let $d_{\partial \Omega_{2}^{\prime}}$ be the distance function to $\partial \Omega_{2}^{\prime},>0$ in $\Omega_{2}^{\prime}$, negative outside. Let $d_{\partial \Omega_{2}^{\prime}, n}=\rho_{n} * d_{\partial \Omega_{2}^{\prime}}, \rho_{n}$ being a positive regularizing kernel. Observe that $\left\|\nabla d_{\partial \Omega_{2}^{\prime}, n}\right\|_{\infty} \leq 1$. We may choose $n$ large enough, and $\Omega_{2}$ such that $\overline{\Omega_{2}} \subset \Omega_{2}^{\prime}, \eta(\delta)<d\left(\partial \Omega_{1}, \partial \Omega_{2}\right)<$ 
$2 \eta(\delta), 0<d_{\partial \Omega_{2}^{\prime}, n}<\eta(\delta)$ in $\partial \Omega_{2}$, and $d_{\partial \Omega_{2}^{\prime}, n}>0$ in $\Omega_{2}$. Let $B_{1,2}=\Omega_{1} \backslash \overline{\Omega_{2}}$. Then, using again Withney's extension Theorem, there is a function $R \in$ $C^{1}\left(\overline{B_{1,2}}\right)$ such that $R=\tilde{D}-\delta$ and $\nabla R=\nabla \tilde{D}$ on $\partial \Omega_{1}$, and $R=d_{\partial \Omega_{2}^{\prime}, n}, \nabla R=$ $\nabla d_{\partial \Omega_{2}^{\prime}, n}$ on $\partial \Omega_{2}$. Moreover, $\|\nabla R\|_{\infty}$ is bounded by a constant depending on $\|\tilde{D}\|_{\infty, \partial \Omega_{1}},\left\|d_{\partial \Omega_{2}^{\prime}, n}\right\|_{\infty, \partial \Omega_{2}},\|\nabla \tilde{D}\|_{\infty, \partial \Omega_{1}},\left\|\nabla d_{\partial \Omega_{2}^{\prime}, n}\right\|_{\infty, \partial \Omega_{2}}$ and

$$
\sup _{x \in \partial \Omega_{1}, y \in \partial \Omega_{2}} \frac{\left|\tilde{D}(x)-\delta-d_{\partial \Omega_{2}^{\prime}, n}(y)\right|}{|x-y|} \leq \frac{2 \eta(\delta)}{\eta(\delta)}=2 .
$$

We define $D: \bar{\Omega} \rightarrow \mathbb{R}$ by

$$
\begin{aligned}
& D=\tilde{D} \text { in } \overline{\Omega_{1}}, \\
& D=R+\delta \text { in } B_{1,2}, \\
& D=d_{\partial \Omega_{2}^{\prime}, n}+\delta \text { in } \overline{\Omega_{2}} .
\end{aligned}
$$

Then $D \in C^{1}(\bar{\Omega}), D=0$ on $\partial \Omega, D>0$ on $\Omega$ and $\nabla D(x)=-\nu(x)$ for all $x \in \partial \Omega$.

Proof of Lemma 3.4 We may think that $u$ and $v$ are extended as $B V$ functions in $\mathbb{R}^{N}$ in such a way that

$$
\int_{\partial \Omega}|D u|=\int_{\partial \Omega}|D v|=0
$$

We consider a family of radially symmetric positive mollifiers

$$
\eta_{j}=\frac{1}{\tau_{j}^{N}} \eta\left(\frac{x}{\tau_{j}}\right), \quad \eta \geq 0, \quad \int_{\mathbb{R}^{N}} \eta(x) d x=1, \quad \tau_{j} \downarrow 0+,
$$

and we set

$$
z_{j}=\eta_{j} *\left(v+\frac{u}{j}\right)
$$

Clearly, we have $z_{j} \in C^{1}(\bar{\Omega})$ and obviously we have

$$
z_{j} \rightarrow v \quad \text { in } L^{N /(N-1)}(\Omega)
$$

Also, from (5.2) it follows that

$$
\int_{\Omega} \sqrt{1+\left|D z_{j}(x)\right|^{2}} d x \rightarrow \int_{\Omega} \sqrt{1+|D v(x)|^{2}} d x .
$$

This implies, by the Theorem of convergence of traces for $B V$ functions that

$$
\left.\left.z_{j}\right|_{\partial \Omega} \rightarrow v\right|_{\partial \Omega} \quad \text { in } L^{1}(\partial \Omega) .
$$


By the Theorem of differentiation of measures ([7]), we obtain

$$
D z_{j}(x) \rightarrow \nabla v(x) \quad \lambda_{N} \text { a.e. in } \Omega \text {. }
$$

Indeed, since $D z_{j}=\eta_{j} * D v+\frac{1}{j} \eta_{j} * D u$, this is a consequence of the four following limits

$$
\begin{aligned}
\lim _{j}\left[\eta_{j} * \nabla v\right](x) & =\nabla v(x) \quad \lambda_{N} \text { a.e. in } \Omega, \\
\lim _{j}\left[\eta_{j} *(D v)^{s}\right](x) & =(D v)^{s}(x)=0 \quad \lambda_{N} \text { a.e. in } \Omega, \\
\lim _{j} \frac{1}{j}\left[\eta_{j} * \nabla u\right](x) & =\nabla u(x) \lim _{j} \frac{1}{j}=0 \quad \lambda_{N} \text { a.e. in } \Omega, \\
\lim _{j} \frac{1}{j}\left[\eta_{j} *(D u)^{s}\right](x) & =0 \quad \lambda_{N} \text { a.e. in } \Omega,
\end{aligned}
$$

since $(D u)^{s},(D v)^{s}$ are singular with respect to $\lambda_{N}$ and $|\nabla u(x)|<\infty \lambda_{N}$ a.e. in $\Omega$. In the same way, using the Theorem of differentiation of measures, we have

$$
\begin{aligned}
& \lim _{j}\left[\eta_{j} * \nabla v\right](x)=0 \quad|D v|^{s} \text { a.e. in } \Omega, \\
& \lim _{j}\left[\eta_{j} * \nabla u\right](x)=0 \quad|D v|^{s} \text { a.e. in } \Omega, \\
& \lim _{j}\left[\eta_{j} *(D u)^{s s}\right](x)=0 \quad|D v|^{s} \text { a.e. in } \Omega, \\
& \lim _{j} \frac{1}{j}\left[\eta_{j} *(D u)^{s a}\right](x)=(D u)^{s a}(x) \lim \frac{1}{j}=0 \quad|D v|^{s} \text { a.e. in } \Omega,
\end{aligned}
$$

where $(D u)^{s a},(D u)^{s s}$ denote the absolutely continuous and singular part of $(D u)^{s}$ with respect to $(D v)^{s}$, and we obtain

$$
\lim _{j} \frac{D z_{j}(x)}{\left|D z_{j}(x)\right|}=\lim _{j} \frac{D z_{j}(x)}{\left|\left[\eta_{j} *(D v)^{s}\right]\right|(x)}=\frac{D v}{|D v|}(x) \quad|D v|^{s} \text { a.e. in } \Omega .
$$

Similarly

$$
\lim _{j}\left|D z_{j}(x)\right|=\lim _{j}\left|\left[\eta_{j} *|D v|^{s}\right](x)\right|=\infty \quad|D v|^{s} \text { a.e. in } \Omega .
$$

Next, we prove that for a suitable choice of the numbers $\tau_{j}$ one has

$$
\lim _{j} \frac{D z_{j}(x)}{(1 / j)\left[\eta_{j} *|D u|^{s s}\right](x)}=\frac{D u}{|D u|}(x) \quad|D u|^{s s} \text { a.e.. }
$$

Assuming this, it is easy to prove that

$$
\lim _{j} \frac{D z_{j}(x)}{\left|D z_{j}(x)\right|}=\frac{D u}{|D u|}(x) \quad|D u|^{s s} \text { a.e.. }
$$


Indeed,

$$
\begin{aligned}
D z_{j}= & \eta_{j} * D v(x)+\frac{1}{j} \eta_{j} * D u(x) \\
= & \eta_{j} * \nabla v(x)+\eta_{j} *(D v)^{s}(x)+\frac{1}{j} \eta_{j} * \nabla u(x) \\
& +\frac{1}{j} \eta_{j} *(D u)^{s a}(x)+\frac{1}{j} \eta_{j} *(D u)^{s s}(x) .
\end{aligned}
$$

Since $\eta_{j} * \nabla v(x) \rightarrow 0, \eta_{j} *(D v)^{s}(x) \rightarrow 0, \frac{1}{j} \eta_{j} * \nabla u(x) \rightarrow 0, \frac{1}{j} \eta_{j} *(D u)^{s a}(x) \rightarrow 0$ $|D u|^{s s}$-a.e., we see that (5.19) follows from (5.18). To prove (5.18) we observe that

$$
\frac{D z_{j}(x)}{(1 / j)\left[\eta_{j} *|D u|^{s s}\right](x)}=\frac{\left[\eta_{j} * D v\right](x)}{(1 / j)\left[\eta_{j} *|D u|^{s s}\right](x)}+\frac{\left[\eta_{j} * D u\right](x)}{\left[\eta_{j} *|D u|^{s s}\right](x)}
$$

Since

$$
\frac{\left[\eta_{j} * D u\right](x)}{\left[\eta_{j} *|D u|^{s s}\right](x)} \rightarrow \frac{D u}{|D u|}(x) \quad|D u|^{s s} \text { a.e., }
$$

it is sufficient to prove that

$$
\frac{\left[\eta_{j} * D v\right](x)}{\frac{1}{j}\left[\eta_{j} *|D u|^{s s}\right](x)} \rightarrow 0 \quad|D u|^{s s} \text { a.e.. }
$$

To prove (5.22), we define

$$
a_{\tau}(x)=\frac{\left[\eta_{\tau} * D v\right](x)}{\left[\eta_{\tau} *|D u|^{s s}\right](x)}
$$

Since $D v$ and $|D u|^{s s}$ are mutually singular, then

$$
a_{\tau}(x) \rightarrow 0 \quad|D u|^{s s} \text { a.e.. }
$$

Thus, if we consider the sets

$$
E(\tau, j)=\left\{x \in \Omega:\left|a_{\tau}(x)\right|>\frac{1}{j^{2}}\right\},
$$

for any fixed $j \in \mathbb{N}$ we have

$$
\lim _{\tau \rightarrow 0}|D u|^{s s}(E(\tau, j))=0 .
$$

For each $j \in \mathbb{N}$, there is some $\tau_{j}$ such that

$$
|D u|^{s s}\left(E\left(\tau_{j}, j\right)\right)<\frac{1}{2^{j}},
$$


that is

$$
|D u|^{s s}\left(\left\{x \in \Omega: j\left|a_{\tau}(x)\right|>\frac{1}{j}\right\}\right)<\frac{1}{2^{j}} .
$$

This easily implies that

$$
\lim _{j \rightarrow \infty} j a_{\tau_{j}}(x)=0, \quad|D u|^{s s} \text { a.e.. }
$$

which is exactly (5.22). Moreover, we may choose $\tau_{j}$ such that $\frac{1}{j}\left[\eta_{j} *\right.$ $\left.|D u|^{s s}\right](x) \rightarrow \infty|D u|^{s s}$ a.e.. From this, and (5.18), it follows that

$$
\left|D z_{j}\right|(x) \rightarrow \infty \quad|D u|^{s s} \text { a.e.. }
$$

We observe that up to know we have not used neither the hypothesis on the regularity of $\partial \Omega$ nor the regularity of $g$.

We observe that the functions $z_{j}$ that we have constructed satisfy some of the requirements of the Lemma but not all of them, in particular, (3.6), (3.8), (3.12), (3.13) have yet to be satisfied. For that, we construct suitable correction functions $\sigma_{j}$ and $\rho_{j}$ around the boundary and we shall define

$$
v_{j}=z_{j}+\sigma_{j}+\rho_{j} .
$$

Let $g_{j} \in C^{1}(\partial \Omega)$ be such that $g_{j} \rightarrow g$ in $L^{1}(\partial \Omega)$. We shall construct the sequence of functions $\sigma_{j} \in C^{1}(\bar{\Omega})$ such that

$$
\begin{aligned}
\sigma_{j} & =g_{j}-z_{j} \quad \text { on } \partial \Omega \\
\int_{\Omega}\left|\sigma_{j}\right|^{\frac{N}{N-1}} & \rightarrow 0, \\
\sigma_{j}(x) & =0 \quad \text { if } D(x)>\varepsilon_{j}+\varepsilon_{j}^{2} \\
\int_{\Omega} \psi \cdot D \sigma_{j} & \rightarrow \int_{\partial \Omega} \psi \cdot \nu(g-v) d H^{N-1}
\end{aligned}
$$

for all $\psi \in C\left(\bar{\Omega}, \mathbb{R}^{N}\right)$,

$$
\begin{aligned}
\int_{\Omega}\left|D \sigma_{j}\right| & \rightarrow \int_{\partial \Omega}|v-g| d H^{N-1} \\
\left|D\left(\sigma_{j}+z_{j}\right)(x)\right| & \rightarrow \infty H^{N-1} \text { a.e. in } T=\{x \in \partial \Omega: g(x) \neq v(x)\},
\end{aligned}
$$

and

$$
\frac{D\left(\sigma_{j}+z_{j}\right)(x)}{\left|D\left(\sigma_{j}+z_{j}\right)(x)\right|} \rightarrow \frac{g(x)-v(x)}{|g(x)-v(x)|} \nu(x)
$$

$H^{N-1}$ a.e. in $T=\{x \in \partial \Omega: g(x) \neq v(x)\}$. 


\section{Construction of $\sigma_{j}$.}

For each number $\varepsilon \in\left(0, \varepsilon_{0}\right)$ we consider a function $h_{\varepsilon}(t):[0, \infty) \rightarrow[0, \infty)$ such that

$$
\begin{aligned}
& h_{\varepsilon} \in C^{1}([0, \infty)), \\
& h_{\varepsilon}^{\prime}(t) \leq 0, \quad h_{\varepsilon}^{\prime}(0)=-\frac{1}{\varepsilon}, \\
& h_{\varepsilon}^{\prime}(t) \text { is not decreasing, } \\
& h_{\varepsilon}(0)=1, h_{\varepsilon}(t)=0 \text { for } t \geq \varepsilon+\varepsilon^{2} .
\end{aligned}
$$

Let $\left\{\varepsilon_{n}\right\}_{n=1}^{\infty}$ be a decreasing sequence of numbers such that

$$
2 \varepsilon_{1}<\varepsilon_{0}<1, \quad \lim _{j} \varepsilon_{j}=0 .
$$

Now, let $G \in W^{1,1}(\Omega)$ such that $G_{\mid \partial \Omega}=g$. Since $g_{j} \in C^{1}(\partial \Omega)$, we may consider a function $G_{j} \in C^{1}(\bar{\Omega})$ which is an extension of $g_{j}$. We may assume that $G_{j} \rightarrow G$ in $L^{1}(\Omega)$ and $\int_{\Omega}\left|\nabla G_{j}\right| \rightarrow \int_{\Omega}|\nabla G|$. We define

$$
\sigma_{j}=\left[G_{j}(x)-z_{j}(x)\right] h_{\varepsilon_{j}}(D(x)) .
$$

Clearly, $\sigma_{j} \in C^{1}(\bar{\Omega})$,

$$
\sigma_{j}=g_{j}-z_{j} \text { on } \partial \Omega,
$$

and, if $D(x)>\varepsilon_{j}+\varepsilon_{j}^{2}$, then $h_{\varepsilon_{j}}(D(x))=0$, and, therefore

$$
\sigma_{j}(x)=0 .
$$

Now,

$$
\int_{\Omega}\left|\sigma_{j}\right|^{N /(N-1)}=\int_{\Omega_{2 \varepsilon_{j}}}\left|\sigma_{j}\right|^{N /(N-1)} \leq \int_{\Omega_{2 \varepsilon_{j}}}\left|G_{j}(x)-z_{j}(x)\right|^{N /(N-1)}
$$

where, for any $\varepsilon>0$, we denote

$$
\Omega_{\varepsilon}=\{x \in \Omega: D(x)<\varepsilon\} .
$$

The functions $G_{j}, z_{j}$ being independent of $\varepsilon_{j}$, we may choose $\varepsilon_{j}>0$ small enough such that

$$
\int_{\Omega_{2 \varepsilon_{j}}}\left|G_{j}(x)-z_{j}(x)\right|^{N /(N-1)}<\frac{1}{j} .
$$

Hence

$$
\int_{\Omega}\left|\sigma_{j}\right|^{N /(N-1)} \rightarrow 0 \quad \text { as } j \rightarrow \infty
$$


Let $\psi \in C\left(\bar{\Omega}, \mathbb{R}^{N}\right)$. Since

$$
\nabla \sigma_{j}(x)=\nabla\left(G_{j}-z_{j}\right)(x) h_{\varepsilon_{j}}(D(x))+\left(G_{j}-z_{j}\right)(x) h_{\varepsilon_{j}}^{\prime}(D(x)) \nabla D(x),
$$

we have

$$
\begin{aligned}
\int_{\Omega} \psi(x) \cdot \nabla \sigma_{j}(x) d x= & \int_{\Omega} \psi(x) \cdot\left(\nabla G_{j}(x)-\nabla z_{j}(x)\right) h_{\varepsilon_{j}}(D(x)) d x \\
& +\int_{\Omega}\left(G_{j}(x)-z_{j}(x)\right) \psi(x) \cdot \nabla\left(h_{\varepsilon_{j}}(D(x))\right) d x \\
= & \int_{\Omega_{\varepsilon_{j}+\varepsilon_{j}^{2}}} \psi(x) \cdot\left(\nabla G_{j}(x)-\nabla z_{j}(x)\right) h_{\varepsilon_{j}}(D(x)) d x \\
& +\int_{\Omega_{\varepsilon_{j}+\varepsilon_{j}^{2}}}\left(G_{j}(x)-z_{j}(x)\right) \psi(x) \cdot h_{\varepsilon_{j}}^{\prime}(D(x)) \nabla D(x) d x
\end{aligned}
$$

Again, since $\left|h_{\varepsilon}\right| \leq 1$ for all $\varepsilon>0$, a proper choice of $\varepsilon_{j}$ guarantees that

$$
\int_{\Omega_{\varepsilon_{j}+\varepsilon_{j}^{2}}} \psi(x) \cdot\left(\nabla G_{j}(x)-\nabla z_{j}(x)\right) h_{\varepsilon_{j}}(D(x)) d x \rightarrow 0
$$

as $j \rightarrow \infty$. Now, by our choice of $G_{j},(5.5)$ and a proper choice of $\varepsilon_{j}$, we have that

$$
\int_{\Omega_{\varepsilon_{j}+\varepsilon_{j}^{2}}}\left(G_{j}(x)-z_{j}(x)\right) \psi(x) \cdot h_{\varepsilon_{j}}^{\prime}(D(x)) \nabla D(x) d x \rightarrow \int_{\partial \Omega} \psi \cdot \nu(g-v) d H^{N-1} .
$$

Indeed, using the change of variable's formula ([13], p. 118, [20], p. 96),

$$
\begin{aligned}
\int_{\Omega_{\varepsilon_{j}+\varepsilon_{j}^{2}}} & \left(G_{j}(x)-z_{j}(x)\right) \psi(x) \cdot h_{\varepsilon_{j}}^{\prime}(D(x)) \nabla D(x) d x \\
= & \int_{0}^{\varepsilon_{j}+\varepsilon_{j}^{2}} \int_{[D=\lambda]}\left(G_{j}(y)-z_{j}(y)\right) \psi(y) \cdot h_{\varepsilon_{j}}^{\prime}(D(y)) \frac{\nabla D(y)}{|\nabla D(y)|} d H^{N-1}(y) d \lambda \\
= & \left(\varepsilon_{j}+\varepsilon_{j}^{2}\right) \int_{\left[D=\lambda_{j}\right]}\left(G_{j}(y)-z_{j}(y)\right) \psi(y) \cdot h_{\varepsilon_{j}}^{\prime}(D(y)) \frac{\nabla D(y)}{|\nabla D(y)|} d H^{N-1}(y)
\end{aligned}
$$

for some $\lambda_{j} \in\left(0, \varepsilon_{j}+\varepsilon_{j}^{2}\right)$ by the intermediate value Theorem. Now, since $G_{j}, z_{j}$ do not depend on our choice of $\varepsilon_{j}$, by choosing $\varepsilon_{j} \rightarrow 0+$ sufficiently fast, we obtain(5.33). Hence

$$
\int_{\Omega} \psi(x) \cdot \nabla \sigma_{j}(x) d x \rightarrow \int_{\partial \Omega} \psi \cdot \nu(g-v) d H^{N-1}
$$


as $j \rightarrow \infty$. In particular, we have

$$
\liminf _{j} \int_{\Omega}\left|\nabla \sigma_{j}(x)\right| d x \geq \int_{\partial \Omega}|g-v| d H^{N-1} .
$$

On the other hand, we have

$$
\begin{aligned}
\int_{\Omega}\left|\nabla \sigma_{j}(x)\right| d x \leq & \int_{\Omega_{\varepsilon_{j}+\varepsilon_{j}^{2}}}\left|\nabla G_{j}(x)-\nabla z_{j}(x)\right| d x \\
& +\int_{\Omega_{\varepsilon_{j}+\varepsilon_{j}^{2}}}\left|G_{j}(x)-z_{j}(x)\right|\left|h_{\varepsilon_{j}}^{\prime}(D(x))\right||\nabla D(x)| d x .
\end{aligned}
$$

Again, a suitable choice of $\varepsilon_{j}$ guarantees that

$$
\int_{\Omega_{\varepsilon_{j}+\varepsilon_{j}^{2}}}\left|\nabla G_{j}(x)-\nabla z_{j}(x)\right| d x \rightarrow 0
$$

as $j \rightarrow \infty$. Similarly, the properties of $G_{j}, z_{j}$ and a choice of $\varepsilon_{j}$ imply that

$$
\int_{\Omega_{\varepsilon_{j}+\varepsilon_{j}^{2}}}\left|G_{j}(x)-z_{j}(x)\right|\left|h_{\varepsilon_{j}}^{\prime}(D(x))\right||\nabla D(x)| d x \rightarrow \int_{\partial \Omega}|g(x)-v(x)| d H^{N-1} .
$$

Hence

$$
\limsup _{j} \int_{\Omega}\left|\nabla \sigma_{j}(x)\right| d x \leq \int_{\partial \Omega}|g(x)-v(x)| d H^{N-1} .
$$

This, together with (5.34) proves that

$$
\lim _{j} \int_{\Omega}\left|\nabla \sigma_{j}(x)\right| d x=\int_{\partial \Omega}|g-v| d H^{N-1} .
$$

Finally, since

$$
D \sigma_{j}+D z_{j}=\nabla G_{j} h_{\varepsilon_{j}}(D)+\nabla z_{j}\left(1-h_{\varepsilon_{j}}(D)\right)+\left(G_{j}-z_{j}\right) h_{\varepsilon_{j}}^{\prime}(D) \nabla D
$$

we may write on $\partial \Omega$

$$
D \sigma_{j}+D z_{j}=\nabla G_{j}-\left(G_{j}-z_{j}\right) h_{\varepsilon_{j}}^{\prime}(0) \nu(x) .
$$

Hence, on $\partial \Omega$, we have

$$
\frac{D \sigma_{j}+D z_{j}}{\left|D \sigma_{j}+D z_{j}\right|}=\frac{\nabla G_{j}-\left(G_{j}-z_{j}\right) h_{\varepsilon_{j}}^{\prime}(0) \nu}{\left|\nabla G_{j}-\left(G_{j}-z_{j}\right) h_{\varepsilon_{j}}^{\prime}(0) \nu\right|}=\frac{\varepsilon_{j} \nabla G_{j}+\left(G_{j}-z_{j}\right) \nu}{\left|\varepsilon_{j} \nabla G_{j}+\left(G_{j}-z_{j}\right) \nu\right|} .
$$


Now, choosing $\varepsilon_{j}$ such that $\varepsilon_{j} \nabla G_{j} \rightarrow 0$ as $j \rightarrow \infty$, we obtain that

$$
\frac{D \sigma_{j}+D z_{j}}{\left|D \sigma_{j}+D z_{j}\right|} \rightarrow \frac{g(x)-v(x)}{|g(x)-v(x)|} \nu(x)
$$

$H^{N-1}$ a.e. in $T=\{x \in \partial \Omega: g(x) \neq v(x)\}$. Next, a proper choice of $\varepsilon_{j}$ in (5.36) guarantees that

$$
\left|D \sigma_{j}(x)+D z_{j}(x)\right| \rightarrow \infty
$$

$H^{N-1}$ a.e. in $T$.

Next, we construct a sequence of functions $\rho_{j} \in C^{1}(\bar{\Omega})$ such that

$$
\begin{array}{rlrl}
\rho_{j} & =0 & \text { on } \partial \Omega, \\
\int_{\Omega}\left|\rho_{j}\right|^{\frac{N}{N-1}} & \rightarrow 0, & \\
\rho_{j}=0 & \text { if } D(x)>\delta_{j}^{2}
\end{array}
$$

for some $\delta_{j}>0$,

$$
\int_{\Omega}\left|D \rho_{j}\right| \rightarrow 0
$$

for $H^{N-1}$-a.e. $x \in T$, there is $j_{0}(x)$ such that

$$
D \rho_{j}(x)=0 \quad \text { for all } j \geq j_{0}(x)
$$

$$
\text { if we set } v_{j}=z_{j}+\sigma_{j}+\rho_{j} \text { then (3.13) holds. }
$$

\section{Construction of $\rho_{j}$.}

For all $\delta>0$ consider a function $\psi_{\delta}:[0, \infty) \rightarrow[0, \infty)$ such that

$$
\left\{\begin{array}{l}
\psi_{\delta} \in C^{1}([0, \infty)) \\
\psi_{\delta}(0)=0, \quad \psi_{\delta}(t)=0 \text { for } t \geq \delta^{2} \\
\left|\psi_{\delta}^{\prime}(t)\right| \leq \frac{4}{\delta}, \text { for } t \in\left(0, \delta^{2}\right) \\
\psi_{\delta}^{\prime}(0) \geq \frac{1}{\delta} \\
\int_{0}^{\infty}\left|\psi_{\delta}^{\prime}(t)\right| d t \leq 2 \delta
\end{array}\right.
$$


We define $\zeta: \partial \Omega \rightarrow \mathbb{R}$

$$
\zeta(x)= \begin{cases}\frac{u(x)-g(x)}{|u(x)-g(x)|} & \text { if } g(x)=v(x) \text { and } g(x) \neq u(x) \\ 0 & \text { elsewhere }\end{cases}
$$

Let $\zeta_{j}$ be a sequence of functions in $C^{1}(\partial \Omega)$ converging to $\zeta$ in $L^{1}(\partial \Omega)$. Now, we may assume that $\zeta$ is the trace of a function $\Theta \in W^{1,1}(\Omega)$ and $\zeta_{j}$ are traces of functions $\Theta_{j} \in C^{1}(\bar{\Omega})$ such that $\Theta_{j} \rightarrow \Theta$ in $L^{1}(\Omega)$ and $\int_{\Omega}\left|D \Theta_{j}\right| \rightarrow \int_{\Omega}|D \Theta|$. Let $\delta_{j}$ be a decreasing sequence of positive numbers that converges to 0 and consider the functions

$$
\rho_{j}(x)=\Theta_{j}(x) \psi_{\delta_{j}}(D(x))
$$

Clearly, $\rho_{j} \in C^{1}(\bar{\Omega}), \rho_{j}(x)=0$ if $x \in \partial \Omega$. Also (5.41) holds. Since, by our choice of the functions $\psi_{\delta}$, we have

$$
\left|\psi_{\delta}(t)\right| \leq 2 \delta
$$

Now,

$$
\int_{\Omega}\left|\rho_{j}\right|^{N /(N-1)} \leq 2 \delta_{j} \int_{\Omega}\left|\Theta_{j}\right|^{N /(N-1)}
$$

which tends to 0 as $j \rightarrow \infty$, which proves (5.40).

Our purpose now is to choose the functions $\zeta_{j}$ such that (5.43) holds. For that, we consider the sets

$$
\begin{gathered}
N^{+}=\{x \in \partial \Omega: \zeta(x)=1\}, \\
N^{-}=\{x \in \partial \Omega: \zeta(x)=-1\}, \\
N=N^{+} \cup N^{-} .
\end{gathered}
$$

We consider increasing sequences of compact sets $K_{j}^{+} \subseteq N^{+}, K_{j}^{-} \subseteq N^{-}$ such that

$$
\lim _{j} H^{N-1}\left(N^{+} \backslash K_{j}^{+}\right)=\lim _{j} H^{N-1}\left(N^{-} \backslash K_{j}^{-}\right)=0 .
$$

We consider also decreasing sequences of open sets $G_{j}^{+} \supseteq N^{+}, G_{j}^{-} \supseteq N^{-}$ such that

$$
\lim _{j} H^{N-1}\left(G_{j}^{+} \backslash N^{+}\right)=\lim _{j} H^{N-1}\left(G_{j}^{-} \backslash N^{-}\right)=0 .
$$

Now, we take functions $\zeta_{j}^{+}, \zeta_{j}^{-} \in C^{1}(\partial \Omega)$ with values in $[0,1]$ such that

$$
\zeta_{j}^{+}(x)= \begin{cases}1 & \text { in } K_{j}^{+} \\ 0 & \text { in } \partial \Omega \backslash G_{j}^{+}\end{cases}
$$




$$
\zeta_{j}^{-}(x)= \begin{cases}1 & \text { in } K_{j}^{-} \\ 0 & \text { in } \partial \Omega \backslash G_{j}^{-}\end{cases}
$$

and we set

$$
\zeta_{j}=\zeta_{j}^{+}-\zeta_{j}^{-}
$$

The functions $\zeta_{j}$ satisfy

$$
\zeta_{j}(x)= \begin{cases}1 & \text { in } K_{j}^{+} \backslash G_{j}^{-} \\ 0 & \text { in } \partial \Omega \backslash\left(G_{j}^{+} \cup G_{j}^{-}\right) \\ -1 & \text { in } K_{j}^{-} \backslash G_{j}^{+}\end{cases}
$$

Moreover

$$
\lim _{j} H^{N-1}\left(T \cap\left(G_{j}^{+} \cup G_{j}^{-}\right)\right)=0 .
$$

Recall that the functions $\Theta_{j}$ are extensions of $\zeta_{j}$ to $\Omega$. Now,

$$
\nabla \rho_{j}(x)=\nabla \Theta_{j}(x) \psi_{\delta_{j}}(D(x))+\Theta_{j}(x) \psi_{\delta_{j}}^{\prime}(D(x)) \nabla D(x) .
$$

If $x \in \partial \Omega$, then

$$
\nabla \rho_{j}(x)=-\Theta_{j}(x) \psi_{\delta_{j}}^{\prime}(0) \nu(x) .
$$

Now, using (5.51), for almost all $x \in T=\{x \in \partial \Omega: g(x) \neq v(x)\}$, there exists $j_{0}(x) \in \mathbb{N}$ such that $\zeta_{j}(x)=0$ for all $j \geq j_{0}(x)$. Hence, also

$$
\nabla \rho_{j}(x)=0 \text { for all } j \geq j_{0}(x) \text {. }
$$

Next

$$
\begin{aligned}
\int_{\Omega}\left|\nabla \rho_{j}\right| & \leq 2 \delta_{j} \int_{\Omega}\left|\nabla \Theta_{j}\right|+\left\|\Theta_{j}\right\|_{\infty}\|\nabla D\|_{\infty} \int_{\Omega_{\delta_{j}^{2}}}\left|\psi_{\delta_{j}}^{\prime}(D(x))\right| d x \\
& \leq 2 \delta_{j} \int_{\Omega}\left|\nabla \Theta_{j}\right|+\left\|\Theta_{j}\right\|_{\infty}\|\nabla D\|_{\infty} \frac{4}{\delta_{j}} \int_{\Omega_{\delta_{j}^{2}}} d x .
\end{aligned}
$$

Now, for $j$ large enough

$$
\int_{\Omega_{\delta_{j}^{2}}} d x=\int_{0}^{\delta_{j}^{2}} \int_{[D=\lambda]} \frac{d H^{N-1}(z)}{|\nabla D(z)|} d \lambda \leq 2 \int_{0}^{\delta_{j}^{2}} \int_{[D=\lambda]} d H^{N-1}(z) d \lambda \leq C \delta_{j}^{2}
$$

where $C$ depends on $\operatorname{Per}(\partial \Omega)$. Hence

$$
\int_{\Omega}\left|\nabla \rho_{j}\right| \leq 2 \delta_{j} \int_{\Omega}\left|\nabla \Theta_{j}\right|+\left\|\Theta_{j}\right\|_{\infty}\|\nabla D\|_{\infty} 4 C \delta_{j} .
$$


Now, choosing $\delta_{j}$ we may guarantee that

$$
\int_{\Omega}\left|\nabla \rho_{j}\right| \rightarrow 0 \quad \text { as } \quad j \rightarrow \infty
$$

Since

$\nabla v_{j}=\nabla G_{j} h_{\varepsilon_{j}}+\nabla z_{j}\left(1-h_{\varepsilon_{j}}\right)+\left(G_{j}-z_{j}\right) h_{\varepsilon_{j}}^{\prime}(D) \nabla D+\nabla \Theta_{j} \psi_{\delta_{j}}+\Theta_{j} \psi_{\delta_{j}}^{\prime} \nabla D$, on $\partial \Omega$, we have

$$
\nabla v_{j}(x)=\nabla G_{j}(x)-\left(G_{j}(x)-z_{j}(x)\right) h_{\varepsilon_{j}}^{\prime}(0) \nu(x)-\Theta_{j}(x) \psi_{\delta_{j}}^{\prime}(0) \nu(x)
$$

and we may write on $\partial \Omega$

$$
\begin{aligned}
\frac{\nabla v_{j}}{\left|\nabla v_{j}\right|} & =\frac{\nabla G_{j}-\left(G_{j}-z_{j}\right) h_{\varepsilon_{j}}^{\prime}(0) \nu-\Theta_{j} \psi_{\delta_{j}}^{\prime}(0) \nu}{\left|\nabla G_{j}-\left(G_{j}-z_{j}\right) h_{\varepsilon_{j}}^{\prime}(0) \nu-\Theta_{j} \psi_{\delta_{j}}^{\prime}(0) \nu\right|}= \\
& =\frac{\delta_{j} \nabla G_{j}-\delta_{j}\left(G_{j}-z_{j}\right) h_{\varepsilon_{j}}^{\prime}(0) \nu-\delta_{j} \Theta_{j} \psi_{\delta_{j}}^{\prime}(0) \nu}{\left|\delta_{j} \nabla G_{j}-\delta_{j}\left(G_{j}-z_{j}\right) h_{\varepsilon_{j}}^{\prime}(0) \nu-\delta_{j} \Theta_{j} \psi_{\delta_{j}}^{\prime}(0) \nu\right|} .
\end{aligned}
$$

Now, we choose $\delta_{j}$ such that $\delta_{j} \nabla G_{j} \rightarrow 0, \delta_{j}\left(G_{j}-z_{j}\right) h_{\varepsilon_{j}}^{\prime}(0) \rightarrow 0$, as $j \rightarrow \infty$, we obtain that

$$
\frac{\nabla v_{j}}{\left|\nabla v_{j}\right|} \rightarrow \frac{g(x)-u(x)}{|g(x)-u(x)|} \nu(x)
$$

$H^{N-1}$ a.e. on $\{x \in \partial \Omega: g(x)=v(x), u(x) \neq v(x)\}$. By choosing $\delta_{j}$ to converge sufficiently fast to 0 , from (5.52), we obtain that

$$
\left|\nabla v_{j}(x)\right| \rightarrow \infty
$$

$H^{N-1}$ a.e. on $\{x \in \partial \Omega: g(x)=v(x), u(x) \neq v(x)\}$.

Let us now check that $v_{j}=z_{j}+\sigma_{j}+\rho_{j}$ satisfies the required properties. Since $v_{j}=g_{j}$ on $\partial \Omega$, (3.6) follows immediately. The property (3.7) follows from (5.4), (5.26) and (5.40). To check (3.8), let $\psi \in C^{1}\left(\bar{\Omega}, \mathbb{R}^{N}\right), \psi=$ $\left(\psi_{1}, \ldots, \psi_{n}\right)$ and $\psi_{N+1} \in C^{1}(\bar{\Omega}, \mathbb{R})$. Using (3.6) and (3.7), we obtain

$$
\begin{aligned}
\lim _{j} \int_{\Omega}[ & \left.\sum_{i=1}^{N} \psi_{i}(x) D_{i} v_{j}(x)+\psi_{N+1}(x)\right] d x \\
& =-\lim _{j} \int_{\Omega} \operatorname{div} \psi(x) v_{j}(x) d x+\int_{\partial \Omega} g_{j} \psi \cdot \nu d H^{N-1}+\int_{\Omega} \psi_{N+1}(x) d x \\
& =-\int_{\Omega} \operatorname{div} \psi(x) v(x) d x+\int_{\partial \Omega} g \psi \cdot \nu d H^{N-1}+\int_{\Omega} \psi_{N+1}(x) d x \\
& =\int_{\Omega}\left[\psi(x) \cdot D v(x)+\psi_{N+1}(x)\right] d x+\int_{\partial \Omega}(g-v) \psi \cdot \nu d H^{N-1}
\end{aligned}
$$


Now, because of the lower semicontinuity of the total variation with respect to weak convergence, we have

$$
\int_{\Omega} \sqrt{1+|D v|^{2}}+\int_{\partial \Omega}|g-v| d H^{N-1} \leq \liminf _{j} \int_{\Omega} \sqrt{1+\left|D v_{j}\right|^{2}} d x
$$

On the other hand, since

$$
\int_{\Omega} \sqrt{1+\left|\nabla v_{j}\right|^{2}} d x \leq \int_{\Omega} \sqrt{1+\left|\nabla z_{j}\right|^{2}} d x+\int_{\Omega}\left|\nabla \sigma_{j}\right| d x+\int_{\Omega}\left|\nabla \rho_{j}\right| d x
$$

using (5.5), (5.29) and (5.42) we obtain that

$$
\limsup _{j} \int_{\Omega} \sqrt{1+\left|D v_{j}\right|^{2}} d x \leq \int_{\Omega} \sqrt{1+|D v|^{2}}+\int_{\partial \Omega}|g-v| d H^{N-1} .
$$

This proves (3.8). Now, using (5.7), (5.27) and (5.41) we obtain (3.9). Next, we observe that (3.10) is a consequence of (5.16), (5.27) and (5.41). In the same way, (3.11) is a consequence of (5.19), (5.27) and (5.41). We observe that (3.12) follows from (5.31) and (5.43). Fina lly, (3.13) has already been proved.

Acknowledgements. The first and third authors have been partially supported by the Spanish DGICYT, Project PB98-1442. The second author acknowledges partial support by the TMR European Project "Viscosity Solutions and their Applications", reference FMRX-CT98-0234 and the PNPGC, Project BFM 2000-0962-C02-01. 


\section{References}

[1] Adams, R. A, Sobolev spaces, Academic Press, Amsterdam, 1975.

[2] Ambrosio, L., Fusco, N. and Pallara, D., Functions of Bounded Variation and Free Discontinuity Problems, Oxford Mathematical Monographs, 2000 .

[3] Andreu, F., Ballester, C., Caselles, V. and Mazón, J. M., Minimizing Total Variational Flow, Differential Integral Equations 14 (2001), 321-360.

[4] Andreu, F., Ballester, C., Caselles, V. and Mazón, J. M., The Dirichlet problem for the total variational flow, J. Funct. Anal. 180 (2001), 347-403.

[5] Anzellotti, G., Pairings Between Measures and Bounded Functions and Compensated Compactness, Ann. Mat. Pura Appl. (4) 135 (1983), 293-318.

[6] Anzellotti, G., The Euler equation for functionals with linear growth, Trans. Amer. Math. Soc. 290 (1985), 483-500.

[7] Anzellotti, G., BV solutions of quasilinear PDEs in divergent form, Comm. Partial Differential Equations 12 (1987), 77-122.

[8] Bernstein, S., Sur les équations du calcul des variations, Ann. Sci. École Norm. Sup. 29 (1912), 431-485.

[9] Brezis, H. Operateurs Maximaux Monotones, North Holland, Amsterdam, 1973.

[10] Bruck, R. E., Asymmptotic convergence of nonlinear contraction semigroups in Hilbert spaces. J. Funct. Anal. 18 (1975), 15-26.

[11] Delfour, M. C. And Zolesio, J. P. , Shape Analysis via Oriented Distance Functions, J. Funct. Anal. 123 (1994), 129-201.

[12] Demengel, F. and Teman, R., Convex Functions of a Measure and Applications, Indiana Univ. Math. J. 33 (1984), 673-709.

[13] Evans, L. C. and Gariepy, R. F. , Measure Theory and Fine Properties of Functions. Studies in Advanced Math., CRC Press, 1992.

[14] Hardt, R. And Zhou, X., An evolution problem for linear growth functionals, Commun. Partial Differential Equations 19 (1994), 1879-1907.

[15] Hormander, L. The Analysis of Linear Partial Differential Operators I. Springer-Verlag, 1983.

[16] Kohn, R. And Temam, R., Dual space of stress and strains with application to Hencky plasticity. Appl. Math. Optim. 10 (1983), 1-35.

[17] Lichnewski, A. and Temam, R., Pseudosolutions of the Time Dependent Minimal Surface Problem, J. Differential Equations 30 (1978), 340-364.

[18] Reshetnyak, Yu. G., Weak convergence of completely additive vector functions on a set, Sibirsk. Mat. Zh. 9 (1968), 1386-1394. (Translated)

[19] Rockafellar, R. T., Duality and stability in extremum problem involvimg convex functions. Pacific J. Math. 21 (1967), 167-187. 
[20] Schwartz, L., Analyse IV. Applications a la théorie de la mesure. Hermann, 1993.

[21] Zhou, X., An Evolution Problem for Plastic Antiplanar Shear. Appl. Math. Optim. 25 (1992), 263-285.

[22] Ziemer, W. P., Weakly Differentiable Functions, GTM 120, Springer Verlag, 1989.

Recibido: 26 de mayo de 2000

Fuensanta Andreu

Universitat de Valencia

Dept. de Análisis Matemático

Dr. Moliner 50, 46100 Burjassot, Spain

Fuensanta.Andreu@uv.es

Vicent Caselles

Universitat Pompeu-Fabra

Dept. de Tecnologia

La Rambla 30-32, 08002 Barcelona, Spain

Vicent.Caselles@tecn.upf.es

José M. Mazón

Universitat de Valencia

Dept. de Análisis Matemático

Dr. Moliner 50, 46100 Burjassot, Spain

Mazon@uv.es 